\title{
Floquet engineering of topological localization transitions and mobility edges in one-dimensional non-Hermitian quasicrystals
}

\author{
Longwen Zhou (1) $^{*}$ \\ College of Physics and Optoelectronic Engineering, Ocean University of China, Qingdao, 266100 China
}

(Received 23 June 2021; revised 9 August 2021; accepted 12 August 2021; published 23 August 2021)

\begin{abstract}
Time-periodic driving fields could endow a system with peculiar topological and transport features. In this work, we find dynamically controlled localization transitions and mobility edges in non-Hermitian quasicrystals via shaking the lattice periodically. The driving force dresses the hopping amplitudes between lattice sites, yielding alternate transitions between localized, mobility edge, and extended non-Hermitian quasicrystalline phases. We apply our Floquet engineering approach to five representative models of non-Hermitian quasicrystals, obtain the conditions of photon-assisted localization transitions and mobility edges, and find the expressions of Lyapunov exponents for some models. We further introduce topological winding numbers of Floquet quasienergies to distinguish non-Hermitian quasicrystalline phases with different localization nature. Our discovery thus extend the study of quasicrystals to non-Hermitian Floquet systems, and provide an efficient way of modulating the topological and transport properties of these unique phases.
\end{abstract}

DOI: 10.1103/PhysRevResearch.3.033184

\section{INTRODUCTION}

Floquet engineering has enabled the realization of rich dynamical, topological, and transport phenomena in a broad range of physical settings [1-17]. Some notable examples include the dynamical localization, Floquet topological phase, and discrete time crystal (see Refs. [18-30] for reviews and references therein). These discoveries have broadened the classification of phases of matter in nonequilibrium situations [31-33], and may lead to applications in emerging technologies such as ultrafast electronics [22] and topological quantum computing [34-36].

Non-Hermitian topological matter has been investigated intensively in the past few years [37-42]. Recently, the Floquet approach was also applied to engineer topological phases in non-Hermitian systems. Notably, it was found that the interplay between time-periodic driving fields and gain and loss or nonreciprocal effects could yield topological phases that are unique to non-Hermitian Floquet systems [43-50]. These intriguing phases are characterized by large integer or half-integer winding numbers and degenerate Floquet edge or corner states with real quasienergies [43-49]. Their topological signatures may further be extracted experimentally from the dynamical spin textures and quantized displacements of wave packets [50]. Till now, various kinds of non-Hermitian Floquet topological insulators, superconductors and semimetals with exceptional topological properties

\footnotetext{
*zhoulw13@u.nus.edu

Published by the American Physical Society under the terms of the Creative Commons Attribution 4.0 International license. Further distribution of this work must maintain attribution to the author(s) and the published article's title, journal citation, and DOI.
}

have been found [43-72]. Meanwhile, much less is known when non-Hermitian Floquet systems are subject to more complicated correlation effects, such as disorder, nonlinearity, and many-body interactions.

In this work, we employ the idea of Floquet engineering to induce and control the spectrum, localization, mobility edges, and topological transitions in non-Hermitian quasicrystals. A quasicrystal is a phase of matter with long-range order in the absence of spatial periodicity. It can be realized in a lattice with diagonal or off-diagonal spatially quasiperiodic modulations. In Sec. II, we outline the method of adjusting hopping amplitudes in a tight-binding lattice by applying high-frequency periodic driving forces, which follows the idea of engineering dynamical localization in ultracold atoms [1-3]. In Sec. III, we introduce five prototypical models of non-Hermitian quasicrystal, which can be viewed as non-Hermitian extensions of the Aubry-AndréHarper (AAH) model and the Maryland model. We further apply high-frequency shaking forces to these models, and obtain driving-induced transitions between non-Hermitian Floquet quasicrystalline phases with distinct transport and topological features. Different types of non-Hermitian quasicrystal phases are also found to emerge alternately with the increase of the ratio between the amplitude and frequency of the driving field. In Sec. IV, we summarize our results and discuss future perspectives.

\section{METHOD}

In this section, we introduce our scheme of Floquet engineering to control the phase transitions in non-Hermitian quasicrystals, which is based on the idea of realizing dynamical localization in Bose-Einstein condensates [19]. A schematic illustration of our system and approach is shown in Fig. 1. The Hamiltonian of the system describes a 


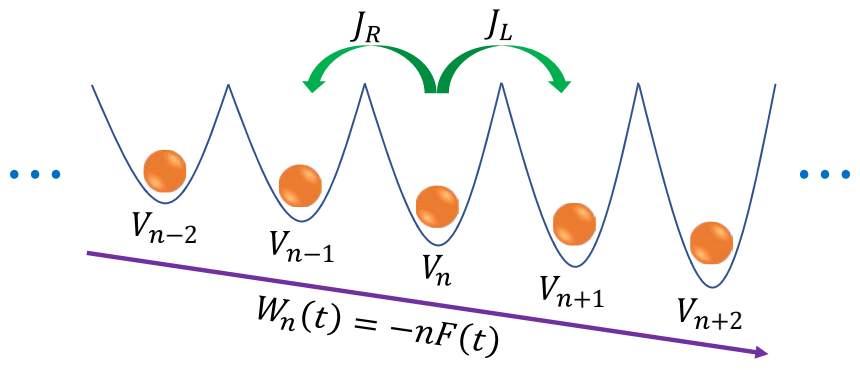

FIG. 1. A schematic illustration of the driven non-Hermitian superlattice. $J_{L}\left(J_{R}\right)$ denotes the hopping amplitude from left (right) to right (left) lattice sites. $V_{n}$ denotes the static potential on the $n$th lattice site, which introduces quasiperiodicity into the system. $W_{n}(t)$ is the driving potential, which is proportional to the position index $n$. The driving force $F(t)$ oscillates periodically in time. The system is made non-Hermitian if $J_{L} \neq J_{R}^{*}$ or $V_{n} \neq V_{n}^{*}$, corresponding to the case with nonreciprocal hopping or on-site gain and loss.

tight-binding superlattice with on-site quasiperiodic potential and driving field, i.e.,

$$
\hat{H}(t)=\sum_{\left\langle n, n^{\prime}\right\rangle}\left(J_{R} \hat{c}_{n}^{\dagger} \hat{c}_{n^{\prime}}+J_{L} \hat{c}_{n^{\prime}}^{\dagger} \hat{c}_{n}\right)+\sum_{n}\left[V_{n}+W_{n}(t)\right] \hat{c}_{n}^{\dagger} \hat{c}_{n},
$$

where $n$ is the lattice site index, and $\left\langle n, n^{\prime}\right\rangle$ includes site indices with $n^{\prime}>n$. $\hat{c}_{n}^{\dagger}$ and $\hat{c}_{n}$ denote the creation and annihilation operators of a particle (either boson or fermion) on the $n$th lattice site. $J_{L}\left(J_{R}\right)$ represents the hopping amplitude of the particle from lattice site $n\left(n^{\prime}\right)$ to $n^{\prime}(n)$. A quasicrystal model is realized by setting the superlattice potential $V_{n}$ to a quasiperiodic function of the lattice index $n$. A non-Hermitian quasicrystal is further obtained by setting $J_{L} \neq J_{R}^{*}$ (for nonreciprocal hopping) or $V_{n} \neq V_{n}^{*}$ (for gain and loss of particles). The coupling between the driving field and the particle takes the form of $W_{n}(t)=-n F(t)$, where the driving force $F(t)=$ $F(t+T)$ varies periodically in time with the driving period $T$ and driving frequency $\omega=2 \pi / T$. All system parameters have been properly rescaled and given in dimensionless units. We will also set the Planck constant $\hbar=1$ throughout the discussion.

In the frame comoving with the lattice, the dynamics of the system is governed by the time-dependent Schrödinger equation

$$
i \frac{d}{d t}|\psi(t)\rangle=\hat{H}(t)|\psi(t)\rangle
$$

The driving potential in $\hat{H}(t)$ can be transformed to an oscillating phase factor in the hopping amplitudes $J_{L, R}$ [19]. To do so, we consider the following unitary rotation:

$$
\hat{R}(t)=e^{i \sum_{n} f_{n}(t) \hat{c}_{n}^{\dagger} \hat{c}_{n}}
$$

where the time-dependent amplitude

$$
\begin{aligned}
f_{n}(t) & =-\int_{t_{0}}^{t} W_{n}\left(t^{\prime}\right) d t^{\prime}-n \bar{f} \\
& =n\left(\int_{t_{0}}^{t} F\left(t^{\prime}\right) d t^{\prime}-\bar{f}\right) \equiv n f(t) .
\end{aligned}
$$

Here $\bar{f}$ is chosen to ensure that the one-period integral of amplitude $\int_{0}^{T} f_{n}(t) d t=0$. Writing the time-evolving state as $|\psi(t)\rangle=\hat{R}(t)\left|\psi^{\prime}(t)\right\rangle$, we obtain the Schrödinger equation for the state $\left|\psi^{\prime}(t)\right\rangle$ in the rotating frame as

$$
i \frac{d}{d t}\left|\psi^{\prime}(t)\right\rangle=\hat{H}_{\mathrm{r}}(t)\left|\psi^{\prime}(t)\right\rangle,
$$

where the Hamiltonian $\hat{H}_{\mathrm{r}}(t)$ in the rotating frame reads

$$
\hat{H}_{\mathrm{r}}(t)=\hat{R}^{\dagger}(t) \hat{H}(t) \hat{R}(t)-i \hat{R}^{\dagger}(t) \frac{d \hat{R}(t)}{d t} .
$$

Plugging Eqs. (3) and (4) into Eq. (6), we find that $\hat{R}(t)$ commutes with the on-site terms $\sum_{n}\left[V_{n}+W_{n}(t)\right] \hat{c}_{n}^{\dagger} \hat{c}_{n}$ in $\hat{H}(t)$ and

$$
i \hat{R}^{\dagger}(t) \frac{d \hat{R}(t)}{d t}=\sum_{n} W_{n}(t) \hat{c}_{n}^{\dagger} \hat{c}_{n},
$$

which cancels the driving potential $\sum_{n} W_{n}(t) \hat{c}_{n}^{\dagger} \hat{c}_{n}$, leaving $\sum_{n} V_{n} \hat{c}_{n}^{\dagger} \hat{c}_{n}$ as the only on-site term in $\hat{H}_{\mathrm{r}}(t)$. Furthermore, the hopping terms in $\hat{H}(t)$ under the rotation $\hat{R}(t)$ becomes

$$
\begin{aligned}
\hat{R}^{\dagger}(t) \hat{c}_{n}^{\dagger} \hat{c}_{n^{\prime}} \hat{R}(t)= & e^{-i n f(t) \hat{c}_{n}^{\dagger} \hat{c}_{n}} \hat{c}_{n}^{\dagger} e^{i n f(t) \hat{c}_{n}^{\dagger} \hat{c}_{n}} \\
& \times e^{-i n^{\prime} f(t) \hat{c}_{n^{\prime}}^{\dagger} \hat{c}_{n^{\prime}}} \hat{c}_{n^{\prime}} e^{i n^{\prime} f(t) \hat{c}_{n^{\prime}}^{\dagger} \hat{c}_{n^{\prime}}} \\
= & e^{i\left(n^{\prime}-n\right) f(t)} \hat{c}_{n}^{\dagger} \hat{c}_{n^{\prime}} .
\end{aligned}
$$

Note that this result holds for both bosons and fermions. Therefore, the Hamiltonian in the rotating frame is

$$
\begin{aligned}
\hat{H}_{\mathrm{r}}(t)= & \sum_{\left\langle n, n^{\prime}\right\rangle} J_{L} e^{i\left(n-n^{\prime}\right) f(t)} \hat{c}_{n^{\prime}}^{\dagger} \hat{c}_{n} \\
& +\sum_{\left\langle n, n^{\prime}\right\rangle} J_{R} e^{i\left(n^{\prime}-n\right) f(t)} \hat{c}_{n}^{\dagger} \hat{c}_{n^{\prime}}+\sum_{n} V_{n} \hat{c}_{n}^{\dagger} \hat{c}_{n},
\end{aligned}
$$

where the oscillating phase factor $f(t)=\int_{t_{0}}^{t} F\left(t^{\prime}\right) d t^{\prime}-\bar{f}$. When $f(t)$ varies quickly in time, so that $\hbar \omega \gg\left|J_{R, L}\right|$ and $\left|V_{n}-V_{n^{\prime}}\right|$ for $\left|n-n^{\prime}\right|=1$, its effect on $\hat{H}_{\mathrm{r}}(t)$ can be approximated by the average over a complete driving period $T$ [19]. This corresponds to taking the zeroth-order term in the highfrequency expansion of the Floquet Hamiltonian [73-75]. In this limit, the micromotion operator is approximated by the rotating frame transformation $\hat{R}(t)$, and the resulting Floquet effective Hamiltonian reads

$$
\begin{aligned}
\hat{H}_{\mathrm{F}} & \approx \frac{1}{T} \int_{0}^{T} d t \hat{H}_{\mathrm{r}}(t) \\
& =\sum_{\left\langle n, n^{\prime}\right\rangle}\left(J_{L}^{\mathrm{eff}} \hat{c}_{n^{\prime}}^{\dagger} \hat{c}_{n}+J_{R}^{\mathrm{eff}} \hat{c}_{n}^{\dagger} \hat{c}_{n^{\prime}}\right)+\sum_{n} V_{n} \hat{c}_{n}^{\dagger} \hat{c}_{n},
\end{aligned}
$$

where the photon-dressed hopping amplitudes $J_{L}^{\text {eff }}$ and $J_{R}^{\text {eff }}$ are given by

$$
\begin{aligned}
& J_{L}^{\mathrm{eff}}=\frac{1}{T} \int_{0}^{T} d t J_{L} e^{i\left(n-n^{\prime}\right) f(t)}, \\
& J_{R}^{\mathrm{eff}}=\frac{1}{T} \int_{0}^{T} d t J_{R} e^{i\left(n^{\prime}-n\right) f(t)} .
\end{aligned}
$$

The driving field thus introduces a control knob to the hopping amplitudes, and thus the transport property of the non-Hermitian quasicrystal. 
In experiments, one type of driving force that is relatively easy to engineer has the form of a harmonic function $[1,19]$, which can be introduced by shaking the lattice back and forth periodically [76-78] and described as $F(t)=K \cos (\omega t)$, where $K$ is the driving amplitude and $\omega$ is the driving frequency of the force. The factor $f(t)$ in the Peierls phase for such a harmonic drive reads $f(t)=K \sin (\omega t) / \omega$ according to Eq. (4). Working out the integrals in Eqs. (11) and (12) with the help of the Bessel function expansion $e^{i x \sin (y)}=$ $\sum_{l=-\infty}^{\infty} \mathcal{J}_{l}(x) e^{i l y}$, we find $J_{L, R}^{\text {eff }}=J_{L, R} \mathcal{J}_{0}\left[\left(n^{\prime}-n\right) K / \omega\right]$, which is derived previously in the study of dynamical localization [76]. The Floquet effective Hamiltonian of the system under the high-frequency harmonic forcing now takes the form

$$
\begin{aligned}
\hat{H}_{\mathrm{F}}= & \sum_{\left\langle n, n^{\prime}\right\rangle} J_{L} \mathcal{J}_{0}\left(\left(n^{\prime}-n\right) \frac{K}{\omega}\right) \hat{c}_{n^{\prime}}^{\dagger} \hat{c}_{n} \\
& +\sum_{\left\langle n, n^{\prime}\right\rangle} J_{R} \mathcal{J}_{0}\left(\left(n^{\prime}-n\right) \frac{K}{\omega}\right) \hat{c}_{n}^{\dagger} \hat{c}_{n^{\prime}}+\sum_{n} V_{n} \hat{c}_{n}^{\dagger} \hat{c}_{n} .
\end{aligned}
$$

Since both the $J_{L, R}$ and the Bessel function of first kind $\mathcal{J}_{0}\left[\left(n^{\prime}-n\right) K / \omega\right]$ decays globally with the increase of hopping distance $\left|n^{\prime}-n\right|$, we may reserve the hopping terms up to nearest-neighbor sites, yielding the following Floquet effective Hamiltonian

$$
\hat{H}_{\mathrm{F}}=\sum_{n}\left[\mathcal{J}_{0}\left(\frac{K}{\omega}\right)\left(J_{R} \hat{c}_{n}^{\dagger} \hat{c}_{n+1}+J_{L} \hat{c}_{n+1}^{\dagger} \hat{c}_{n}\right)+V_{n} \hat{c}_{n}^{\dagger} \hat{c}_{n}\right] .
$$

In the Hermitian limit, we observe that the hopping amplitudes $J_{L, R}$ are switched off at the zeros of $\mathcal{J}_{0}(K / \omega)$, which leads to the well-known phenomena of dynamical localization for any nonvanishing on-site potential $V_{n}$ [1]. When the system becomes non-Hermitian and $V_{n}$ is quasiperiodic, the competition between the energy scales of intersite tunneling and on-site trapping could result in localization-delocalization transitions caused by non-Hermitian effects. The presence of the driving field further generates an interplay between the field parameters $(K, \omega)$ and the non-Hermitian terms of the system, which is expected to yield richer phase diagrams and dynamically controlled transitions between different nonHermitian quasicrystalline phases. These judgments will be demonstrated by explicit examples in the following section.

\section{RESULTS}

In this section, we apply the Floquet engineering approach discussed in Sec. II to five representative models of non-Hermitian quasicrystal, which can be viewed as nonHermitian variants of the AAH model [79-81] and the Maryland model [82-85]. The explicit forms of these models and the driving forces are introduced in Sec. III A. Without the driving field, the conditions of localization transition for these models have been derived in previous studies. We reveal how these conditions are modified in the presence of high-frequency lattice shaking forces, and obtain analytical expressions for their Lyapunov exponents if possible, which are now functions of the driving amplitude and frequency. Our theoretical results are further verified by numerical calculations presented in Secs. III B-III F.

\section{A. Quasicrystal models}

The AAH model is one of the standard models in the study of localization-delocalization transitions in one-dimensional (1D) quasiperiodic systems [79-81]. In the lattice representation, the Hamiltonian of the model takes the form

$$
\hat{H}_{0}=\sum_{n}\left[J\left(\hat{c}_{n}^{\dagger} \hat{c}_{n+1}+\text { H.c. }\right)+V \cos (2 \pi \alpha n) \hat{c}_{n}^{\dagger} \hat{c}_{n}\right],
$$

where $J$ is the hopping amplitude and $V$ is the amplitude of the on-site potential. By setting $\alpha$ to be an irrational number, the superlattice potential $V \cos (2 \pi \alpha n)$ becomes quasiperiodic in the lattice index $n$, and the system described by $\hat{H}_{0}$ forms a $1 \mathrm{D}$ quasicrystal. Thanks to the self-duality property of the AAH quasicrystal, it has been shown that the eigenstates of $\hat{H}_{0}$ possess a delocalization-to-localization transition at $V=2 J$ in the thermodynamic limit. When $V<2 J$, all eigenstates of $\hat{H}_{0}$ are delocalized and the system is in an extended phase. When $V>2 J$, the correlated disorder becomes strong enough, such that all eigenstates of $\hat{H}_{0}$ are localized and the system enters an insulating phase [81].

The Maryland model forms another paradigm in the study of Anderson localization and quantum chaos [82-85]. It is an integrable model and can be mapped to a Floquet system. The Hamiltonian of the Maryland model takes the form

$$
\hat{H}_{\mathrm{M}}=\sum_{n}\left[J\left(\hat{c}_{n}^{\dagger} \hat{c}_{n+1}+\text { H.c. }\right)+V \tan (\pi \alpha n) \hat{c}_{n}^{\dagger} \hat{c}_{n}\right],
$$

where $n$ is the lattice index, $J$ is the hopping amplitude, $V$ is the amplitude of the on-site potential, and a quasicrystal is formed once $\alpha$ takes an irrational value. Since the potential $V_{n}$ depends on a tangent function, which is not bounded, all states of the system are localized and are found to have an energy-dependent Lyapunov exponent (inverse localization length) $\lambda(E)=\operatorname{arccosh}\left[\left(\sqrt{(2 J+E)^{2}+V^{2}}+\right.\right.$ $\left.\left.\sqrt{(2 J-E)^{2}+V^{2}}\right) / 4|J|\right][82]$. Besides, the Maryland model can also be employed to study the topological nature of integer quantum Hall effects [86].

In recent years, the impact of non-Hermiticity on localization transitions in AAH-type and Maryland-type quasicrystals have been explored [84,87-107]. The non-Hermitian effects are introduced by either setting the on-site quasiperiodic potential to be non-Hermitian [84,87-89], or making the hopping amplitudes between adjacent lattice sites to be nonreciprocal $[90,91]$. In these models, it was found that the non-Hermitian terms could induce $\mathcal{P} \mathcal{T}$ transitions of the energy spectrum from real to complex (or the opposite), together with localization-delocalization transitions of the eigenstates. These transitions could further be characterized by the quantized change of winding numbers of the spectrum around certain base points of the complex energy plane [84,87-91].

In the following sections, we apply the Floquet engineering approach to induce and control phase transitions in five non-Hermitian quasicrystal models. The time-dependent Hamiltonians of these models take the general form of Eq. (1), with the explicit expressions of hopping amplitudes, on-site potential, and driving force given in Table I. We will refer to these five models as M1-M5 for simplicity. In the highfrequency limit, the Floquet effective Hamiltonians of these models then take the formalism of Eq. (14), where the hopping 
TABLE I. Definitions of the five periodically forced non-Hermitian quasicrystal models, denoted by M1-M5. In the second column, $J_{L}$ and $J_{R}$ are left-to-right and right-to-left hopping amplitudes between nearest-neighbor lattice sites. $\gamma$ controls the asymmetry between the left and right hopping amplitudes in M3. In the third column, $V$ is the amplitude of the on-site potential, $i \gamma$ is an imaginary phase shift, $\eta$ controls the non-Hermiticity of M5 and $\alpha$ is an irrational number. In the fourth column, $n$ is the lattice site index, $K$ is the driving amplitude and $\omega$ is the driving frequency. All system parameters are set in dimensionless units.

\begin{tabular}{|c|c|c|c|}
\hline $\begin{array}{l}\text { Model } \\
\text { index }\end{array}$ & $\begin{array}{l}\text { Hopping } \\
\text { amplitude }\end{array}$ & $\begin{array}{c}\text { On-site } \\
\text { potential } V_{n}\end{array}$ & $\begin{array}{c}\text { Driving } \\
\text { force } W_{n}(t)\end{array}$ \\
\hline M1 & $J_{L}=J_{R}=J$ & $V e^{-i 2 \pi \alpha n}$ & $-n K \cos (\omega t)$ \\
\hline M2 & & $V \cos (2 \pi \alpha n+i \gamma)$ & \\
\hline M3 & $J_{L}=J e^{\gamma}, J_{R}=J e^{-\gamma}$ & $V \cos (2 \pi \alpha n)$ & \\
\hline M4 & $J_{L}=J_{R}=J$ & $V \tan (\pi \alpha n+i \gamma)$ & \\
\hline M5 & & $V /\left(1-\eta e^{i 2 \pi \alpha n}\right)$ & \\
\hline
\end{tabular}

amplitudes are controlled by the ratio of the driving amplitude and driving frequency $K / \omega$ through the Bessel function $\mathcal{J}_{0}(K / \omega)$. Since the localization-delocalization transitions in these models are originated from the competition between the energy scales of hopping $J$ and onsite potential $V$ [81], the photon-dressed hopping amplitude $J_{L, R} \mathcal{J}_{0}(K / \omega)$ provides a flexible knob to tune the transitions between different nonHermitian quasicrystalline phases of these systems, as will be demonstrated below. Note in passing that the models M1-M5 do not possess the translation symmetry. They also do not have the chiral symmetry due to the absence of sublattice or spin structures. Meanwhile, the effective Hamiltonians of M1-M5 all possess the $\mathcal{P} \mathcal{T}$ symmetry, which is relevant for the characterization of their phase transitions.

\section{B. M1: Floquet spectrum, localization transition, and topological invariant}

Following Table I, the time-dependent Hamiltonian of the quasicrystal M1 reads

$$
\begin{aligned}
\hat{H}_{1}(t)= & \sum_{n} J\left(\hat{c}_{n}^{\dagger} \hat{c}_{n+1}+\text { H.c. }\right) \\
& +\sum_{n}\left[V e^{-i 2 \pi \alpha n}-n K \cos (\omega t)\right] \hat{c}_{n}^{\dagger} \hat{c}_{n},
\end{aligned}
$$

which is non-Hermitian due to the complex on-site potential $V e^{-i 2 \pi \alpha n}$ and quasiperiodic when $\alpha$ is irrational. Following the procedure of Sec. II, we find the Floquet effective Hamiltonian of the system under high-frequency driving as

$$
\hat{H}_{1 \mathrm{~F}}=\sum_{n}\left[J \mathcal{J}_{0}\left(\frac{K}{\omega}\right)\left(\hat{c}_{n}^{\dagger} \hat{c}_{n+1}+\text { H.c. }\right)+V e^{-i 2 \pi \alpha n} \hat{c}_{n}^{\dagger} \hat{c}_{n}\right] \text {. }
$$

The hopping strength now depends on the ratio between the amplitude and frequency of the driving field, and can thus be controlled dynamically. Meanwhile, the effective Hamiltonian $\hat{H}_{1 \mathrm{~F}}$ possesses the $\mathcal{P} \mathcal{T}$ symmetry as $V_{n}=V_{-n}^{*}$, which means that its Floquet spectrum could be real in certain parameter regions.

The eigenvalue equation of $\hat{H}_{1 \mathrm{~F}}$ is $\hat{H}_{1 \mathrm{~F}}|\psi\rangle=E|\psi\rangle$. Inserting the single-particle state $|\psi\rangle=\sum_{n} \psi_{n} \hat{c}_{n}^{\dagger}|0\rangle$ into the equation, we obtain the eigenvalue equation in the lattice representation as

$$
J \mathcal{J}_{0}\left(\frac{K}{\omega}\right)\left(\psi_{n+1}+\psi_{n-1}\right)+V e^{-i 2 \pi \alpha n} \psi_{n}=E \psi_{n} .
$$

Here $E \bmod 2 \pi$ corresponds to the quasienergy of $\hat{H}_{1 \mathrm{~F}}$. For a lattice of length $L$ and under the periodic boundary condition (PBC), one can take a discrete Fourier transformation from lattice to momentum representations by expanding the amplitude $\psi_{n}$ as

$$
\psi_{n}=\frac{1}{\sqrt{L}} \sum_{m=1}^{L} \varphi_{m} e^{-i 2 \pi \alpha m n} .
$$

The transformed eigenvalue equation in momentum space then reads

$$
V \varphi_{n-1}+2 J \mathcal{J}_{0}\left(\frac{K}{\omega}\right) \cos (2 \pi \alpha n) \varphi_{n}=E \varphi_{n} .
$$

Following the method outlined in Ref. [89], it can be readily shown that when $\alpha$ takes irrational values, the non-Hermitian Floquet quasicrystal $\mathrm{M} 1$ possesses a $\mathcal{P} \mathcal{T}$ transition and a localization-delocalization transition under the condition

$$
|V|=\left|J \mathcal{J}_{0}(K / \omega)\right| .
$$

When $|V|<\left|J \mathcal{J}_{0}(K / \omega)\right|$, the hopping process dominates and all Floquet eigenstates of $\hat{H}_{1 \mathrm{~F}}$ are extended with vanishing Lyapunov exponents (diverging localization lengths). The quasienergy dispersion of $\hat{H}_{1 \mathrm{~F}}$ in this case takes the form of $E(k)=2 J \mathcal{J}_{0}(K / \omega) \cos (k) \in \mathbb{R}$, where $k \in[0,2 \pi)$. When $|V|>\left|J \mathcal{J}_{0}(K / \omega)\right|$, the quasiperiodic potential dominates, and all Floquet eigenstates of $\hat{H}_{1 \mathrm{~F}}$ are localized with the same Lyapunov exponent (inverse localization length) $\lambda=$ $-\ln \left|J \mathcal{J}_{0}(K / \omega) / V\right|>0$. The Floquet spectrum of the system in this case has the form of $E(k)=2 J \mathcal{J}_{0}(K / \omega) \cos (k-i \lambda) \in$ $\mathbb{C}$, where $k \in[0,2 \pi)$. The extended and localized phases could be further distinguished by a topological winding number of the Floquet spectrum. With the eigenvalue equation (21), we find the matrix elements of the system's Floquet effective Hamiltonian $\hat{H}_{1 \mathrm{~F}}^{\prime}$ in momentum space to be

$$
\begin{aligned}
{\left[\hat{H}_{1 \mathrm{~F}}^{\prime}\right]_{n, n} } & =2 J \mathcal{J}_{0}\left(\frac{K}{\omega}\right) \cos (2 \pi \alpha n), \quad n=1, \ldots, L ; \\
{\left[\hat{H}_{1 \mathrm{~F}}^{\prime}\right]_{n+1, n} } & =\left[\hat{H}_{1 \mathrm{~F}}^{\prime}\right]_{1, L}=V, \quad n=1, \ldots, L-1 .
\end{aligned}
$$

Multiplying the corner matrix element $\left[\hat{H}_{1 \mathrm{~F}}^{\prime}\right]_{1, L}$ by a phase factor $e^{-i \theta}$, we obtain the effective Hamiltonian $\hat{H}_{1 \mathrm{~F}}^{\prime}(\theta)$ under the twist boundary condition in momentum space. The spectral winding number of $\hat{H}_{1 \mathrm{~F}}^{\prime}(\theta)$ can then be obtained following the construction of Ref. [91]. Such a winding number counts the number of times the Floquet spectrum of $\hat{H}_{1 \mathrm{~F}}^{\prime}(\theta)$ winds around 
TABLE II. Summary of the results for the non-Hermitian Floquet quasicrystal M1. $J$ is the nearest-neighbor hopping amplitude, $V$ is the amplitude of on-site non-Hermitian quasiperiodic potential, $K$ is the driving amplitude, $\omega$ is the driving frequency, and $\mathcal{J}_{0}(K / \omega)$ denotes the Bessel function of first kind. $\lambda$ denotes the Lyapunov exponent [108]. The parameter $k$ fills uniformly the range of $[0,2 \pi)$. $\hat{H}_{1 \mathrm{~F}}^{\prime}(\theta)$ is obtained from $\hat{H}_{1 \mathrm{~F}}$ after taking the discrete Fourier transformation and introducing a phase twist $e^{-i \theta}$ to its top-right corner matrix element in momentum representation. $E_{0}$ is the base quasienergy.

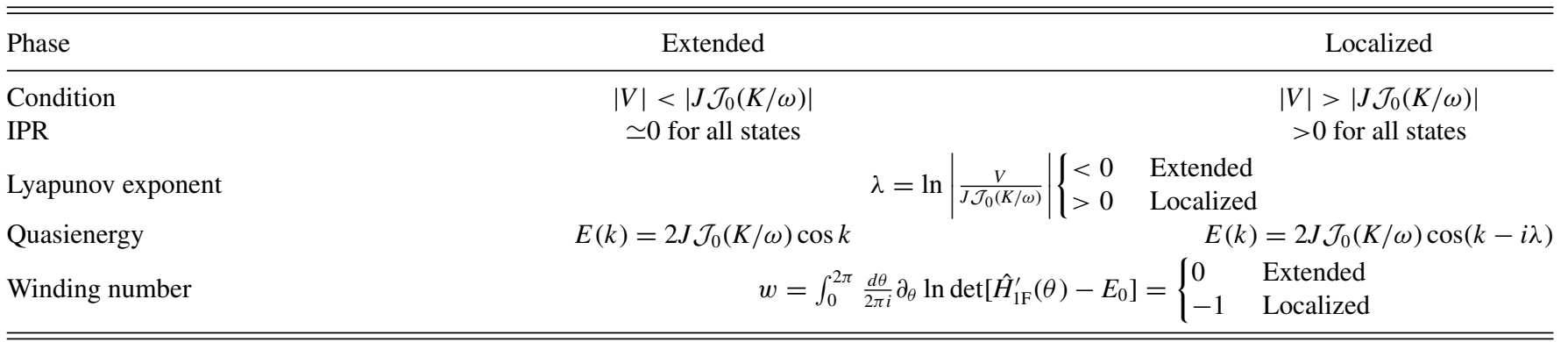

a base quasienergy $E_{0}$ on the complex plane $\operatorname{Re} E-\operatorname{Im} E$ when $\theta$ changes from zero to $2 \pi$. When the quasienergy spectrum is real (complex), the value of this winding number is found to be zero $(-1)$. This winding number could thus serve as a topological order parameter to distinguish the extended phase (with real quasienergies) and localized phase (with complex quasienergies) of the system. In Table II, we summarize the key results about the spectrum, localization transition and topological invariant of the non-Hermitian Floquet quasicrystal M1. Notably, the condition of localization transition in the system now depends on the parameter $K / \omega$ of the driving field. The states of the system can thus be tuned between extended and localized phases dynamically by varying the ratio $K / \omega$ between the amplitude and frequency of the driving force.

To demonstrate our theoretical results, we compute the spectrum $E$, inverse participation ratio (IPR), Lyapunov exponent $\lambda$ and winding number $w$ of the M1 versus the amplitude of onsite potential $V$ and the ratio between driving amplitude and frequency $K / \omega$. The numerical results are presented in Fig. 2. In the calculation, we choose the undressed hopping amplitude $J=1$ and the quasicrystal parameter $\alpha=\frac{\sqrt{5}-1}{2} \simeq \frac{p}{q}$, with $p, q$ being two adjacent elements of the Fibonacci sequence $(p<q)$. The length of lattice is chosen as $L=610$, and the base quasienergy is set as $E_{0}=0$. In Fig. 2(a), we show the maximal imaginary parts of quasienergy $\max |\operatorname{Im} E|$ versus $V$ and $K / \omega$. The red dashed line is given by the phase boundary equation $|V|=$ $\left|J \mathcal{J}_{0}(K / \omega)\right|$. It is clear that $\max |\operatorname{Im} E|=0(\max |\operatorname{Im} E|>0)$ when $|V|<\left|J \mathcal{J}_{0}(K / \omega)\right|\left[|V|>\left|J \mathcal{J}_{0}(K / \omega)\right|\right]$. The condition $|V|=\left|J \mathcal{J}_{0}(K / \omega)\right|$ thus determines the boundary of the $\mathcal{P} \mathcal{T}$ transition between real and complex quasienergy spectrum of the system.

For any normalized Floquet right eigenstate $|\psi\rangle=$ $\sum_{n} \psi_{n} \hat{c}_{n}^{\dagger}|0\rangle$ of $\hat{H}_{1 \mathrm{~F}}$, with $\sum_{n=1}^{L}\left|\psi_{n}\right|^{2}=1$, we define its IPR as

$$
\mathrm{IPR}=\sum_{n=1}^{L}\left|\psi_{n}\right|^{4} .
$$

The minimum of IPRs over all eigenstates at a fixed set of system parameters under the PBC then yields the $\min (\mathrm{IPR})$. In Fig. 2(b), we present the $\min (\mathrm{IPR})$ versus $V$ and $K / \omega$, which is found to be $\simeq 0$ when $|V|<\left|J \mathcal{J}_{0}(K / \omega)\right|$ (below the red dashed line) and $>0$ otherwise. The maximum of IPRs of all eigenstates is found to have the same dependence on $V$ and $K / \omega$. Therefore, the system is indeed in the extended (localized) phase with all states being delocalized (localized) when $|V|<\left|J \mathcal{J}_{0}(K / \omega)\right|\left[|V|>\left|J \mathcal{J}_{0}(K / \omega)\right|\right]$. Finally, we present the signs of Lyapunov exponent $\lambda=-\ln \left|J \mathcal{J}_{0}(K / \omega) / V\right|$ and the topological winding numbers $w$ of M1 versus $V$ and $K / \omega$ in Fig. 2(c). It is clear that the winding number $w=0$ in the extended phase with real quasieneriges, and $w=-1$ in the localized phase with complex quasienergies. The two regions are separated by the phase boundary $|V|=\left|J \mathcal{J}_{0}(K / \omega)\right|$ (red dashed line). Therefore, it is verified that $w$ could be utilized as a topological order parameter to discriminate the $\mathcal{P} \mathcal{T}$ invariant extended phase and $\mathcal{P} \mathcal{T}$-breaking localized phase of the non-Hermitian Floquet quasicrystal M1. Note that without
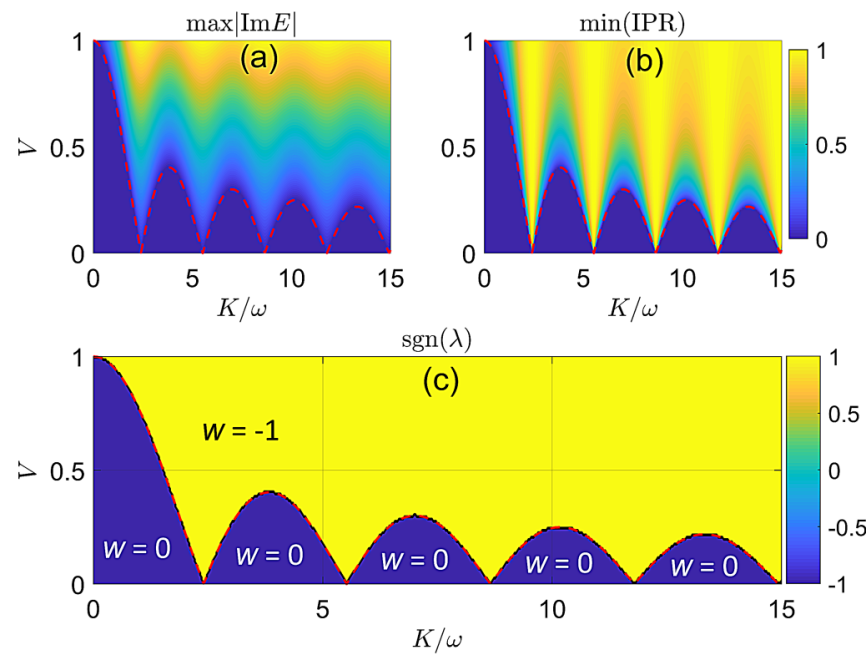

FIG. 2. The maximal imaginary parts of Floquet quasienergies in (a), the minimal values of IPRs in (b), and the signs of Lyapunov exponents in (c) of the non-Hermitian Floquet quasicrystal M1. Other system parameters are set as $\alpha=\frac{\sqrt{5}-1}{2}, J=1$, and the length of lattice is taken as $L=610$. The red dashed line in each figure panel denotes the boundary between the extended phase (with real quasienergies for all Floquet eigenstates) and the localized phase (with complex quasienergies for all Floquet eigenstates), which satisfies the condition $|V|=\left|J \mathcal{J}_{0}(K / \omega)\right|$ (see Table II). The topological winding number $w$ of each phase is denoted explicitly in (c). 
the driving field, the phase boundary between the extended and localized states reduces to a point at $V=J$. The driving force instead allows the localized phase to disappear and reappear alternately with the change of the driving parameter $K / \omega$, which highlights the advantage of Floquet engineering in the realization and control of phase transitions in non-Hermitian quasicrystals.

\section{M2: Floquet spectrum, localization transition, and topological invariant}

We next consider a driven non-Hermitian AAH quasicrystal with an imaginary phase shift $i \gamma$, whose Hamiltonian takes the form of

$$
\begin{aligned}
\hat{H}_{2}(t)= & \sum_{n} J\left(\hat{c}_{n}^{\dagger} \hat{c}_{n+1}+\text { H.c. }\right) \\
& +\sum_{n}[V \cos (2 \pi \alpha n+i \gamma)-n K \cos (\omega t)] \hat{c}_{n}^{\dagger} \hat{c}_{n} .
\end{aligned}
$$

According to the theory presented in Sec. II, the Floquet effective Hamiltonian of this non-Hermitian quasicrystal M2 is given by

$$
\begin{aligned}
\hat{H}_{2 \mathrm{~F}}= & \sum_{n} J \mathcal{J}_{0}\left(\frac{K}{\omega}\right)\left(\hat{c}_{n}^{\dagger} \hat{c}_{n+1}+\text { H.c. }\right) \\
& +\sum_{n} V \cos (2 \pi \alpha n+i \gamma) \hat{c}_{n}^{\dagger} \hat{c}_{n} .
\end{aligned}
$$

It is clear that the hopping amplitude is modified by the driving field and its magnitude could be controlled by the ratio between the amplitude and frequency of the driving force $K / \omega$. Besides, the $\hat{H}_{2 \mathrm{~F}}$ also holds the $\mathcal{P} \mathcal{T}$ symmetry, since its on-site potential $V_{n}=V \cos (2 \pi \alpha n+i \gamma)=V_{-n}^{*}$. The Floquet quasienergy spectrum of $\hat{H}_{2 \mathrm{~F}}$ could then take real values in certain parameter regions.

Plugging the single-particle state $|\psi\rangle=\sum_{n} \psi_{n} \hat{c}_{n}^{\dagger}|0\rangle$ into $\hat{H}_{2 \mathrm{~F}}|\psi\rangle=E|\psi\rangle$, we obtain

$$
J \mathcal{J}_{0}\left(\frac{K}{\omega}\right)\left(\psi_{n+1}+\psi_{n-1}\right)+V \cos (2 \pi \alpha n+i \gamma) \psi_{n}=E \psi_{n},
$$

where $E \bmod 2 \pi$ refers to the quasienergy. Taking the PBC for a finite lattice of length $L$ and performing the discrete Fourier transformation by Eq. (20), we find the eigenvalue equation in momentum space to be

$$
\frac{V}{2}\left(e^{\gamma} \varphi_{n-1}+e^{-\gamma} \varphi_{n+1}\right)+2 J \mathcal{J}_{0}\left(\frac{K}{\omega}\right) \cos (2 \pi \alpha n) \varphi_{n}=E \varphi_{n},
$$

which can also be viewed formally as a quasiperiodic lattice with nonreciprocal hopping and photon-dressed on-site potential.

Following the method developed in Ref. [88], it is straightforward to show that the non-Hermitian Floquet quasicrystal M2 could undergo a $\mathcal{P} \mathcal{T}$ transition and a localizationdelocalization transition when its parameters satisfy the condition

$$
|V| e^{|\gamma|}=\left|2 J \mathcal{J}_{0}(K / \omega)\right|,
$$

which is clearly dependent on the parameters of the driving field $K$ and $\omega$. If $|V| e^{|\gamma|}<\left|2 J \mathcal{J}_{0}(K / \omega)\right|$, the hopping energy overwhelms the quasiperiodic on-site disorder, and the system sits in an extended phase. All Floquet eigenstates in this phase have real quasieneriges and delocalized profiles (with vanishing IPRs) throughout the lattice. If $|V| e^{|\gamma|}>$ $\left|2 J \mathcal{J}_{0}(K / \omega)\right|$, the non-Hermitian on-site potential governs the behavior of the system and drives it into a localized phase. The Floquet states of the system in this phase are all localized (with finite IPRs) and have complex quasienergies. These localized eigenstates are found to share the common Lyapunov exponent $\lambda=-\ln \left|2 J \mathcal{J}_{0}(K / \omega) /\left(V e^{|\gamma|}\right)\right|$, whose value is also controlled by the ratio between the amplitude and frequency of the driving force. To construct a topological winding number for $\mathrm{M} 2$, we add a real phase shift to the quasiperiodic on-site potential [88] by setting $\cos (2 \pi \alpha n+i \gamma) \rightarrow \cos (2 \pi \alpha n+i \gamma+\theta / L)$ in Eq. (26) where $L$ is the length of lattice. We denote the phase-shifted effective Hamiltonian by $\hat{H}_{2 \mathrm{~F}}(\theta)$, and the number of times that its quasienergy spectrum winds around a base point $E_{0}$ on the complex plane defines a topological invariant of the system, which is equal to -1 when the spectrum is complex, and zero otherwise. Therefore, the non-Hermitian Floquet quasicrystal M1 possesses an extended phase with real spectrum and winding number $w=0$, and a localized phase with complex spectrum and winding number $w=-1$. By tuning the ratio $K / \omega$ between the amplitude and frequency of the driving force, the system could also roam alternately between the $\mathcal{P} \mathcal{T}$-invariant extended phase and the $\mathcal{P} \mathcal{T}$-breaking localized phase. The topological and transport properties of the non-Hermitian quasicrystal M2 could thus be controlled by the periodic driving field. We summarize the key results about the spectrum, phases, and topological features of M2 in Table III.

To verify our theoretical predictions, we compute the quasienergy spectrum, IPR, Lyapunov exponent, and winding number of the non-Hermitian Floquet quasicrystal M2 numerically, with results reported in Fig. 3. In the calculation, we choose the system parameters $(J, V)=(2,1)$ and the quasiperiodic parameter $\alpha=\frac{\sqrt{5}-1}{2} \simeq \frac{p}{q}$, where $p, q$ are two neighboring elements of the Fibonacci sequence $(p<q)$. The length of lattice is set as $L=610$, and the base quasienergy is chosen to be $E_{0}=0$. In Fig. 3(a), we show the maximum of the imaginary parts of Floquet spectrum versus the parameter of driving force $K / \omega$ and imaginary part of phase shift $\gamma$. The red dashed line highlights the boundary of $\mathcal{P} \mathcal{T}$ transition, which is determined by Eq. (29). The numerical results confirm that the Floquet spectrum of the system is indeed real when $|V| e^{|\gamma|}<\left|2 J \mathcal{J}_{0}(K / \omega)\right|$ (below the red dashed line), and complex in other circumstances. In Fig. 3(b), we show the minimum of IPRs, which behaves similarly as the maximum of IPRs versus $K / \omega$ and $\gamma$ at each given set of system parameters. The results indicate that the IPRs of all states approach zero when $|V| e^{|\gamma|}<\left|2 J \mathcal{J}_{0}(K / \omega)\right|$ (below the red dashed line), and become finite if $|V| e^{|\gamma|}>\left|2 J \mathcal{J}_{0}(K / \omega)\right|$. Therefore, the condition in Eq. (29) defines the boundary 
TABLE III. Summary of the results for the non-Hermitian Floquet quasicrystal M2. $J$ is the nearest-neighbor hopping amplitude, $V$ is the amplitude of onsite potential, $\gamma$ is the imaginary part of superlattice phase shift, $K$ is the driving amplitude, $\omega$ is the driving frequency, and $\mathcal{J}_{0}(K / \omega)$ denotes the Bessel function of first kind. $\lambda$ denotes the Lyapunov exponent [108]. $H_{2 \mathrm{~F}}(\theta)$ is obtained from $H_{2 \mathrm{~F}}$ after setting $V_{n}=V \cos (2 \pi \alpha n+i \gamma+\theta / L)$, with $L$ being the length of lattice. $E_{0}$ is the base quasienergy.

\begin{tabular}{|c|c|c|c|c|c|}
\hline Phase & Extended & & & & Localized \\
\hline Condition & $|V| e^{|\gamma|}<\left|2 J \mathcal{J}_{0}(K / \omega)\right|$ & & & & $|V| e^{|\gamma|}>\left|2 J \mathcal{J}_{0}(K / \omega)\right|$ \\
\hline IPR & $\simeq 0$ for all states & & & & $>0$ for all states \\
\hline Lyapunov exponent & $\lambda=\ln$ & $\frac{V e^{|\gamma|}}{2 J \mathcal{J}_{0}(K / \omega)} \mid$ & $\begin{cases}<0 & \mathrm{E} \\
>0 & \mathrm{~L}\end{cases}$ & $\begin{array}{l}\text { Extended } \\
\text { Localized }\end{array}$ & \\
\hline Quasienergy & Real & & & & Complex \\
\hline Winding number & $w=\int_{0}^{2 \pi} \frac{d \theta}{2 \pi i} \partial_{\theta} \ln$ & $\operatorname{det}\left[\hat{H}_{2 \mathrm{~F}}\right.$ & $\left.-E_{0}\right]=$ & $=\left\{\begin{array}{l}0 \\
-1\end{array}\right.$ & $\begin{array}{l}\text { Extended } \\
\text { Localized }\end{array}$ \\
\hline
\end{tabular}

between the $\mathcal{P} \mathcal{T}$-invariant extended phase and the $\mathcal{P} \mathcal{T}$ breaking localized phase of the non-Hermitian Floquet quasicrystal M2. Finally, we show the signs of Lyapunov exponent $\lambda$ and the topological winding numbers $w$ versus $K / \omega$ and $\gamma$ in Fig. 3(c), in which we observe $\lambda<0(\lambda>0)$ for all states in the extended (localized) phase with $w=0(w=-1)$, as shown by the blue (yellow) regions below (above) the phase boundary [i.e., the red dashed line satisfying Eq. (29)]. Therefore, the winding number $w$ works as a topological invariant to distinguish the $\mathcal{P} \mathcal{T}$-breaking localized phase and $\mathcal{P} \mathcal{T}$-invariant extended phase of the system. Similar to the case of M1, the driving force now allows one to change the system over a series of transitions between localized and delocalized phases. This further demonstrates the generality of our Floquet engineering approach to the design and control of non-Hermitian quasicrystalline phases in different model systems.
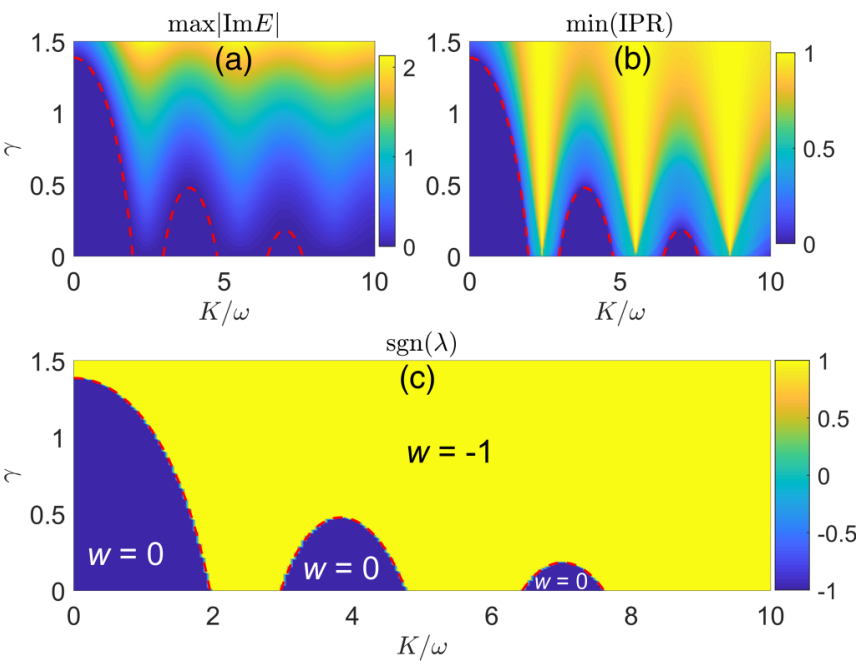

FIG. 3. The maximal imaginary parts of Floquet quasienergies in (a), the minimal values of IPRs in (b), and the signs of Lyapunov exponents in (c) of the non-Hermitian Floquet quasicrystal M2. Other system parameters are set as $\alpha=\frac{\sqrt{5}-1}{2}, J=2, V=1$, and the length of lattice is taken as $L=610$. The red dashed line in each figure panel denotes the boundary between the extended phase (with real quasienergies for all Floquet eigenstates) and the localized phase (with complex quasienergies for all states), which satisfies the condition $|V| e^{|\gamma|}=\left|J \mathcal{J}_{0}(K / \omega)\right|$ (see Table III). The topological winding number $w$ of each phase is denoted explicitly in (c).

\section{M3: Floquet spectrum, localization transition, and topological invariant}

In the third part of this section, we consider a nonreciprocal variant of the AAH model [90,91], which is subject to a periodically modulated driving force. The time-dependent Hamiltonian of the model takes the form

$$
\begin{aligned}
\hat{H}_{3}(t)= & \sum_{n} J\left(e^{-\gamma} \hat{c}_{n}^{\dagger} \hat{c}_{n+1}+e^{\gamma} \hat{c}_{n+1}^{\dagger} \hat{c}_{n}\right) \\
& +\sum_{n}[V \cos (2 \pi \alpha n)-n K \cos (\omega t)] \hat{c}_{n}^{\dagger} \hat{c}_{n},
\end{aligned}
$$

which is non-Hermitian if $\gamma \neq 0$. Following the steps of Sec. II, we obtain the Floquet effective Hamiltonian of this non-Hermitian quasicrystal M3 under the high-frequency approximation as

$$
\begin{aligned}
\hat{H}_{3 \mathrm{~F}}= & \sum_{n} \mathcal{J}_{0}\left(\frac{K}{\omega}\right) J\left(e^{-\gamma} \hat{c}_{n}^{\dagger} \hat{c}_{n+1}+e^{\gamma} \hat{c}_{n+1}^{\dagger} \hat{c}_{n}\right) \\
& +\sum_{n} V \cos (2 \pi \alpha n) \hat{c}_{n}^{\dagger} \hat{c}_{n} .
\end{aligned}
$$

The hopping amplitude is now controlled by the amplitude $K$ and frequency $\omega$ of the driving field. Applying $\hat{H}_{3 \mathrm{~F}}$ to the eigenstate $|\psi\rangle=\sum_{n} \psi_{n} \hat{c}_{n}^{\dagger}|0\rangle$, we further obtain the eigenvalue equation in lattice representation

$$
\mathcal{J}_{0}\left(\frac{K}{\omega}\right) J\left(e^{\gamma} \psi_{n-1}+e^{-\gamma} \psi_{n+1}\right)+V \cos (2 \pi \alpha n) \psi_{n}=E \psi_{n} .
$$

That this equation takes the same form as Eq. (28) under the exchange of system parameters $V \leftrightarrow 2 J \mathcal{J}_{0}(K / \omega)$, which implies that the non-Hermitian Floquet quasicrystals M2 and M3 are dual to each other concerning their spectrum and states. Furthermore, taking the Fourier transformation of Eq. (32) with the help of Eq. (20), we obtain

$$
\frac{V}{2}\left(\varphi_{n+1}+\varphi_{n-1}\right)+2 J \mathcal{J}_{0}\left(\frac{K}{\omega}\right) \cos (2 \pi \alpha n-i \gamma) \varphi_{n}=E \varphi_{n},
$$

which again shares the same form with Eq. (27). Putting together, we find that under the $\mathrm{PBC}$, there is indeed a duality relation between the $\mathrm{M} 2$ and $\mathrm{M} 3$, in the sense that the localization properties of the M2 in momentum space is consistent with the localization properties of the M3 in position space 
TABLE IV. Summary of results for the non-Hermitian Floquet quasicrystal M3. $J$ is the symmetric part of nearest-neighbor hopping amplitude, $\gamma$ controls the asymmetry between left and right hopping amplitudes, $V$ is the amplitude of on-site quasiperiodic potential, $K$ is the driving amplitude, $\omega$ is the driving frequency, and $\mathcal{J}_{0}(K / \omega)$ denotes the Bessel function of first kind. $\lambda$ denotes the Lyapunov exponent [108]. $H_{3 \mathrm{~F}}(\theta)$ is obtained from $H_{3 \mathrm{~F}}$ after taking the periodic boundary condition and setting its top-right (bottom-left) corner matrix element to be $J_{L} \mathcal{J}_{0}(K / \omega) e^{-i \theta}\left[J_{R} \mathcal{J}_{0}(K / \omega) e^{i \theta}\right] . E_{0}$ is the base quasienergy.

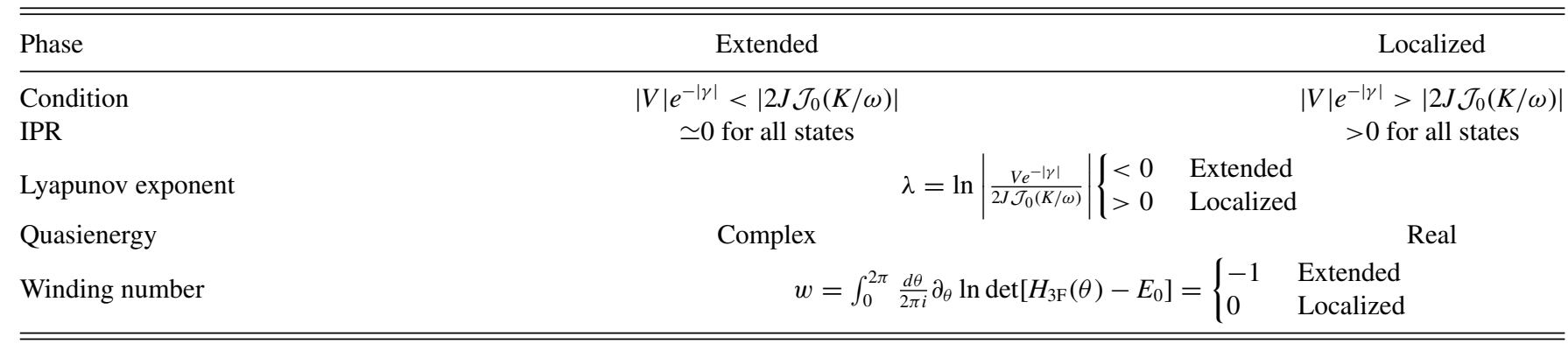

under the PBC. With the help of this duality relation, we can immediately write down the phase boundary condition that determines the $\mathcal{P} \mathcal{T}$ transition and localization-delocalization transition in the M3 under the PBC, i.e.,

$$
|V|=\left|2 J \mathcal{J}_{0}(K / \omega)\right| e^{|\gamma|} .
$$

In parallel with the discussions of Sec. IIIC, if $|V|<$ $\left|2 J \mathcal{J}_{0}(K / \omega)\right| e^{|\gamma|}$, the M3 is found to be in a $\mathcal{P} \mathcal{T}$-breaking extended phase (dual to the localized phase of M2) with complex quasienergies and vanishing IPRs for all Floquet eigenstates. When $|V|>\left|2 J \mathcal{J}_{0}(K / \omega)\right| e^{|\gamma|}$, the $\mathrm{M} 3$ is in a $\mathcal{P} \mathcal{T}$-invariant localized phase (dual to the extended phase of M2), where all Floquet eigenstates have real quasienergies, finite IPRs, and the same positive Lyapunov exponent $\lambda=$ $-\ln \left|2 J \mathcal{J}_{0}(K / \omega) e^{|\gamma|} / V\right|$ in the thermodynamic limit $L \rightarrow \infty$. Note that due to the dependence of phase boundary condition Eq. (34) and Lyapunov exponent on the ratio between the amplitude and frequency of the driving force $K / \omega$, transitions between phases with different spectrum and transport features in the M3 can also be induced by the driving field.

To define a spectral winding number, we take the twist boundary condition [91] by adding phase factors to the corner matrix elements of $\hat{H}_{3 \mathrm{~F}}$ in the lattice representation, i.e.,

$$
\left[\hat{H}_{3 \mathrm{~F}}\right]_{1, L}=J e^{(\gamma-i \theta)}, \quad\left[\hat{H}_{3 \mathrm{~F}}\right]_{L, 1}=J e^{(i \theta-\gamma)},
$$

where $L$ is the length of lattice and $\theta \in[0,2 \pi)$. The spectrum of the phase-dependent Hamiltonian $\hat{H}_{3 \mathrm{~F}}(\theta)$ could now possess a winding number around certain base quasienergy $E_{0}$ on the complex plane when $\theta$ changes over a cycle from zero to $2 \pi$. Such a winding number could be nonzero only if the spectrum of $\hat{H}_{3 \mathrm{~F}}(\theta)$ is complex. Therefore, we expect a quantized winding number to appear for the $\mathcal{P} \mathcal{T}$-breaking extended phase, and a vanishing one for the $\mathcal{P} \mathcal{T}$-invariant localized phase of the system. Such a winding number can then be used as a topological invariant to distinguish the two nonHermitian Floquet quasicrystalline phases of M3 with distinct spectrum and transport nature. We summarize the main results about the M3 in Table IV for the ease of reference.

To confirm our theoretical predictions, we present the Floquet spectrum, IPR, Lyapunov exponent, and winding number of the M3 versus the imaginary part of phase shift $\gamma$ and the ratio between the amplitude and frequency of driving field $K / \omega$ in Fig. 4. Other system parameters are set as $J=V=1$ in the calculation. The quasicrystal parameter $\alpha=\frac{\sqrt{5}-1}{2} \simeq \frac{p}{q}$, with $p, q$ being adjacent elements of the Fibonacci sequence $(p<q)$. The length of lattice is chosen to be $L=610$, and the base quasienergy is set as $E_{0}=0$. In Figs. 4(a)-4(c), the red dashed lines refer to the phase boundary satisfying the Eq. (34). We observe that the maximum of imaginary parts of quasienergy $\max |\operatorname{Im} E|$ vanishes below the red dashed line and taking finite values above it, as shown in Fig. 4(a). The system thus undergoes a $\mathcal{P} \mathcal{T}$ transition when $|V| e^{-|\gamma|}$ increases from below $\left|2 J \mathcal{J}_{0}(K / \omega)\right|$ to above it, through which the Floquet spectrum changes from real to complex. In Fig. 4(b), we show the minimum of IPRs over all Floquet eigenstates at each given set of system parameters. The maximum of IPRs versus $K / \omega$ and $\gamma$ are found to have the same pattern. Therefore, all states of the system are extended in the $\mathcal{P} \mathcal{T}$-breaking regime (with complex

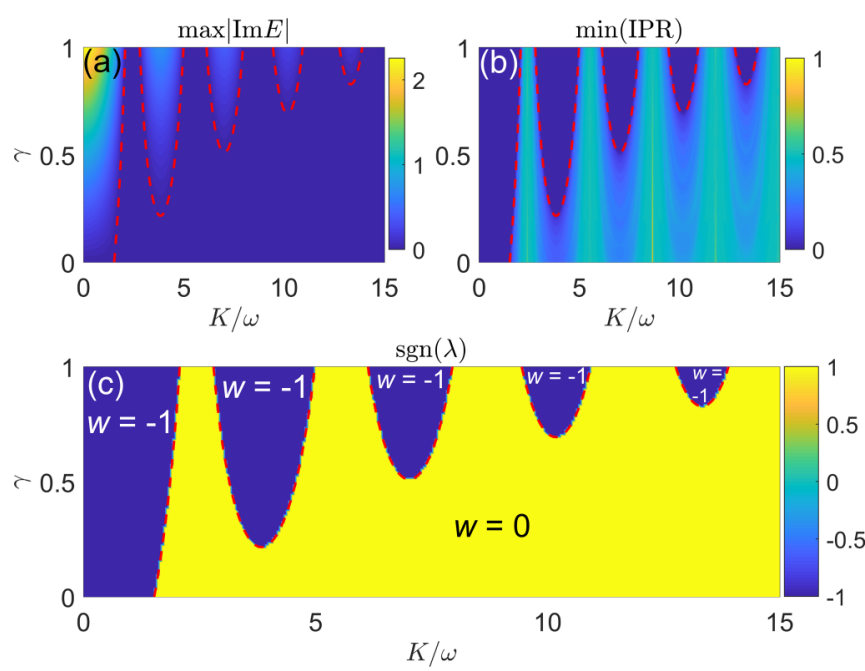

FIG. 4. The maximal imaginary parts of quasienergies in (a), the minimal values of IPRs in (b), and the signs of Lyapunov exponents in (c) of the non-Hermitian Floquet quasicrystal M3. Other system parameters are set as $\alpha=\frac{\sqrt{5}-1}{2}, J=1, V=1$, and the length of lattice is taken as $L=610$. The red dashed line in each figure panel denotes the boundary between the extended phase (with complex quasienergies for all states) and the localized phase (with real quasienergies), which satisfies the condition $|V| e^{-|\gamma|}=\left|J \mathcal{J}_{0}(K / \omega)\right|$ (see Table IV). The topological winding number $w$ of each phase is denoted explicitly in (c). 
spectrum), and localized in the $\mathcal{P} \mathcal{T}$-invariant regime (with real spectrum). In Fig. 4(c), we show the signs of Lyapunov exponent $\operatorname{sgn}(\lambda)$ and winding numbers $w$ of different phases. We obtain identical and positive (negative) $\lambda$ for all states below (above) the phase boundary line, implying a phase with localized (delocalized) states at all quasienergies. Moreover, we find the winding number $w=0$ and $w=-1$ in the localized and delocalized phases, respectively, and a quantized change of $w$ across the phase boundary (red dashed line). The driven non-Hermitian quasicrystal M3 thus possesses a $\mathcal{P} \mathcal{T}$ breaking extended phase with topological winding number $w=-1$, in which all Floquet eigenstates are delocalized with complex quasienergies, and a $\mathcal{P} \mathcal{T}$-invariant localized phase with topological winding number $w=0$, in which all Floquet eigenstates are localized with real quasienergies. As the phase boundary is controlled by the amplitude and frequency of the driving force, the system can also be tuned dynamically to go through transitions between different non-Hermitian quasicrystalline phases following our Floquet engineering scheme.

\section{E. M4: Floquet spectrum, localization transition, mobility edge, and topological invariant}

To further reveal the usefulness of the Floquet engineering scheme, we now apply it to a non-Hermitian quasicrystal that could possess energy-dependent mobility edges. In the lattice representation, the Hamiltonian of the system describes a non-Hermitian extension of the Maryland model [82-84] subjecting to an oscillating force, which takes the form

$$
\begin{aligned}
\hat{H}_{4}(t)= & \sum_{n} J\left(\hat{c}_{n}^{\dagger} \hat{c}_{n+1}+\text { H.c. }\right) \\
& +\sum_{n}[V \tan (\pi \alpha n+i \gamma)-n K \cos (\omega t)] \hat{c}_{n}^{\dagger} \hat{c}_{n} .
\end{aligned}
$$

Following the theory presented in Sec. II, the Floquet effective Hamiltonian of this non-Hermitian quasicrystal M4 reads

$$
\begin{aligned}
\hat{H}_{4 \mathrm{~F}}= & \sum_{n} J \mathcal{J}_{0}\left(\frac{K}{\omega}\right)\left(\hat{c}_{n}^{\dagger} \hat{c}_{n+1}+\text { H.c. }\right) \\
& +\sum_{n} V \tan (\pi \alpha n+i \gamma) \hat{c}_{n}^{\dagger} \hat{c}_{n},
\end{aligned}
$$

in which the fast driving field generates a modulation to the hopping amplitude through the factor $\mathcal{J}_{0}(K / \omega)$. Expressing the Floquet eigenstate as $|\psi\rangle=\sum_{n} \psi_{n} \hat{c}_{n}^{\dagger}|0\rangle$, we obtain the eigenvalue equation

$$
J \mathcal{J}_{0}\left(\frac{K}{\omega}\right)\left(\psi_{n+1}+\psi_{n-1}\right)+V \tan (\pi \alpha n+i \gamma) \psi_{n}=E \psi_{n},
$$

where $E \bmod 2 \pi$ refers to the quasienergy of $|\psi\rangle$.

Following the mapping between the Maryland model and an equivalent Floquet problem [82-84], it can be shown that this non-Hermitian quasicrystal M4 possesses two phase transitions at $\gamma=\gamma_{1}$ and $\gamma=\gamma_{2}$, with

$$
\begin{aligned}
& \gamma_{1}=\frac{1}{2} \operatorname{arcsinh}\left(V /\left|J \mathcal{J}_{0}(K / \omega)\right|\right), \\
& \gamma_{2}=\frac{1}{2} \operatorname{arccosh} \sqrt{\Delta+\sqrt{\Delta^{2}-1}},
\end{aligned}
$$

where $\Delta \equiv\left\{2+\left[V / J \mathcal{J}_{0}(K / \omega)\right]^{2}\right\} / 2$. When the imaginary part of phase shift $\gamma<\gamma_{1}$, all eigenstates of the system are localized with quasienergies

$$
E= \pm \sqrt{\left[2 J \mathcal{J}_{0}(K / \omega) \cos (\beta+i \gamma)\right]^{2}+V^{2} \cot ^{2}(\beta+i \gamma)}
$$

where $\beta \in[0, \pi]$ and $\operatorname{Im} E>0$. These quasienergies form a closed loop around $E_{1}=i V$ on the complex plane, whose size and shape can be controlled by the ratio between the amplitude and frequency of the driving force.

At $\gamma=\gamma_{1}$, the system undergoes a transition from a localized phase to a phase with quasienergy-dependent mobility edges, and stays in this mobility edge phase when $\gamma \in\left(\gamma_{1}, \gamma_{2}\right)$. The spectrum of the system in this phase can be separated into two parts. Within the range of $\left(-2\left|\cos \beta_{0}\right|, 2\left|\cos \beta_{0}\right|\right)$ on the complex energy plane, where

$$
\beta_{0}=\arccos \left(\cos (2 \gamma) \sqrt{1-\frac{V^{2}}{\left[J \mathcal{J}_{0}(K / \omega) \sin (2 \gamma)\right]^{2}}}\right),
$$

the imaginary part of the spectrum is pinned at $V$, and $E$ takes the form of

$$
E=2 J \mathcal{J}_{0}(K / \omega) \cos \beta+i V, \quad \beta \in\left[-\left|\beta_{0}\right|,\left|\beta_{0}\right|\right] .
$$

It is clear that the two ends of the spectrum along the real axis are controlled by the driving field, which could merge into one when $K / \omega$ is tuned to the zeros of the Bessel function $\mathcal{J}_{0}$. Since only the states whose quasienergies satisfy Eq. (43) have delocalized profiles in the mobility edge phase, the driving field provides us with a convenient knob to engineer and control the mobility edges of the non-Hermitian Maryland model. Beyond the range of $\left(-2\left|\cos \beta_{0}\right|, 2\left|\cos \beta_{0}\right|\right)$, the spectrum of the system is still described by Eq. (41), which now arranges into two loops around $E_{2}^{ \pm}= \pm 2 J \mathcal{J}_{0}(K / \omega)+i V$.

At $\gamma=\gamma_{2}$, the system experiences a second transition from the mobility edge phase to an extended phase, in which all eigenstates are delocalized and their quasienergies are described by Eq. (43) for $\gamma>\gamma_{2}$, with $\beta \in[-\pi, \pi$ ). The spectrum now collapses into a line ending at $E_{2}^{ \pm}$, and the loops described by Eq. (41) all vanish. The ending points of the spectrum of delocalized states could again be controlled by the driving field.

Following the Avila's global theory [84,109,110], the system is found to have a quasienergy-dependent Lyapunov exponent $\lambda\left(E=E_{\mathrm{r}}+i E_{\mathrm{i}}\right)$ in the thermodynamic limit, where $E_{\mathrm{r}}$ and $E_{\mathrm{i}}$ are the real and imaginary parts of $E$ [see Table $\mathrm{V}$ for the expression of $\lambda(E)]$. In the extended and localized phases, one has $\lambda(E)=0$ and $\lambda(E)>0$ for all states, respectively. In the mobility edge phase, we have $\lambda(E)=0$ for eigenstates with $E_{\mathrm{r}} \in\left(-2\left|\cos \beta_{0}\right|, 2\left|\cos \beta_{0}\right|\right)$, and $\lambda(E)>0$ otherwise. As expected, the Lyapunov exponents, and therefore the localization nature of the Floquet states can be controlled by the ratio between the amplitude and frequency of the driving field $K / \omega$.

Finally, following the routines of Secs. III B-IIID, we could construct a pair of spectral winding numbers $\left(w_{1}, w_{2}\right)$ to characterize the topological nature of transitions between different phases (see Table $\mathrm{V}$ for their definitions). In the localized phase, both the base energies $E_{1}=i V$ and $E_{2}=$ $2 J \mathcal{J}_{0}(K / \omega)+i V$ are encircled once by the spectrum of 
TABLE V. Summary of results for the non-Hermitian Floquet quasicrystal M4. $J$ is the nearest-neighbor hopping amplitude, $V$ is the amplitude of on-site potential, $\gamma$ is the imaginary part of superlattice phase shift, $K$ is the driving amplitude, $\omega$ is the driving frequency, and $\mathcal{J}_{0}(K / \omega)$ denotes the Bessel function of first kind. The phase variable $\beta \in[-\pi, \pi) . H_{4 \mathrm{~F}}(\theta)$ is obtained from $H_{4 \mathrm{~F}}$ after setting $V_{n}=$ $V \tan (\pi \alpha n+i \gamma+\theta / L)$, with $L$ being the length of lattice. $E_{1}=i V$ and $E_{2}=2 J \mathcal{J}_{0}(K / \omega)+i V$ are two base quasienergies with respect to which the two winding numbers $\left(w_{1}, w_{2}\right)$ are defined.

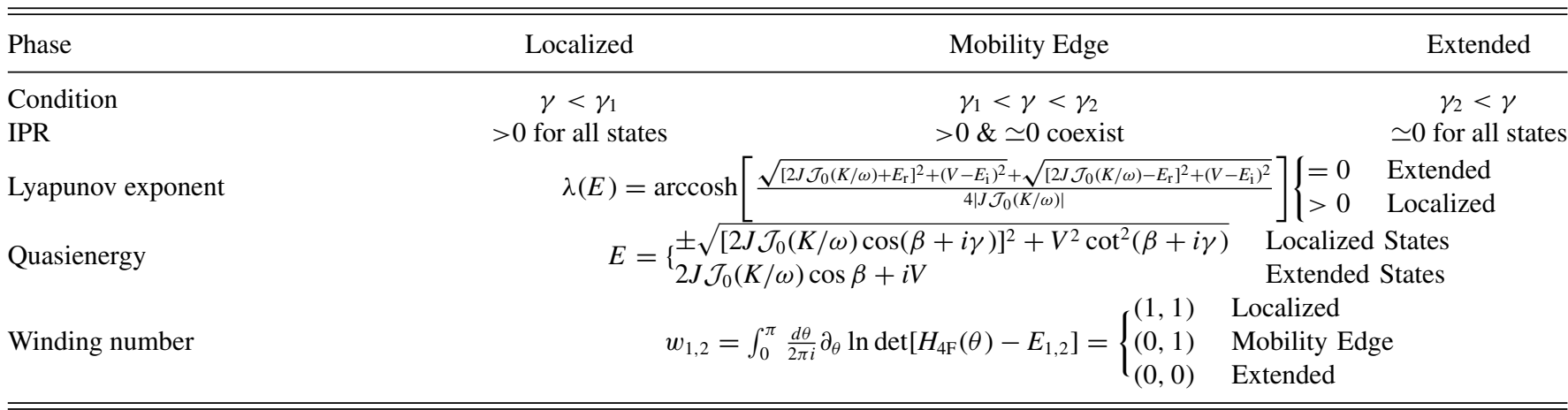

$H_{4 \mathrm{~F}}(\theta)$ when $\theta$ changes over a cycle from zero to $\pi$, and the winding numbers $\left(w_{1}, w_{2}\right)=(1,1)$. In the mobility edge phase, only $E_{2}=2 J \mathcal{J}_{0}(K / \omega)+i V$ is encircled once by the spectral of $H_{4 \mathrm{~F}}(\theta)$ for $\theta \in[0, \pi]$, and the winding numbers $\left(w_{1}, w_{2}\right)=(0,1)$. In the extended phase, none of the base energies $E_{1,2}$ are encircled by the spectral and we have $\left(w_{1}, w_{2}\right)=(0,0)$. Therefore, these winding numbers could be employed to distinguish phases with different transport properties in the M4. For the ease of reference, we summarize the main results of the M4 in Table V.

To verify our theoretical results, we present numerical calculations of the IPRs, Lyapunov exponents, and winding numbers of the non-Hermitian Floquet quasicrystal M4 versus $K / \omega$ and $\gamma$ in Figs. 5 and 6. We find that states with the
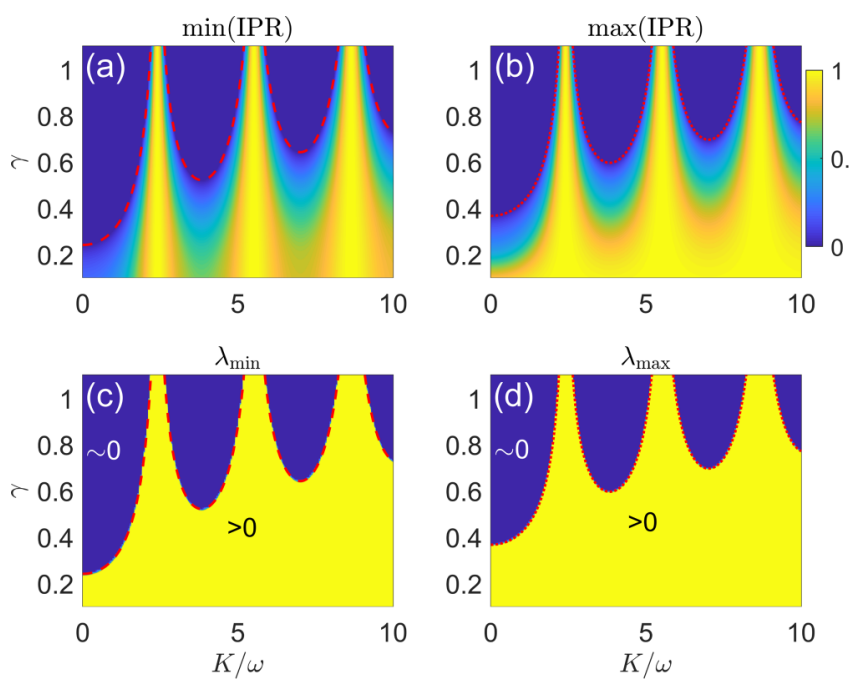

FIG. 5. The minimal (maximal) values of IPRs in (a) [(b)], and the minimum (maximum) of Lyapunov exponents in (c) [(d)] of the non-Hermitian Floquet quasicrystal M4. Other system parameters are set as $\alpha=\frac{\sqrt{5}-1}{2}, J=2, V=1$, and the length of lattice is taken as $L=610$. The red dashed and dotted lines in each figure panel denote the boundaries between the extended phase, mobility edge phase, and localized phase, respectively, which satisfy the conditions in Eqs. (39) and (40). minimum of IPRs $\simeq 0$ and Lyapunov exponent $\lambda_{\min } \simeq 0$ start to emerge when $\gamma$ passes over $\gamma_{1}$ from below, as shown in Figs. 5(a) and 5(c). This means that some states of the system become delocalized after the first transition at $\gamma=\gamma_{1}$ (red dashed line). When $\gamma$ further increases to $\gamma_{2}$ (red dotted line), a second transition happens and all states become extended with $\max (\mathrm{IPR}) \simeq 0$ and $\lambda_{\max } \simeq 0$ after the transition $\left(\gamma>\gamma_{2}\right)$, as shown in Figs. 5(b) and 5(d). The boundaries between the three distinct quasicrystal phases are precisely determined by Eqs. (39) and (40). Besides, the system could also enter and leave the localized, mobility edge and extended phases alternately with the increase of $K / \omega$, and the regime of mobility edge is modified at different values of $K / \omega$. Therefore, both the localization-delocalization transitions and mobility edges in the non-Hermitian Maryland model M4 could be controlled by the driving field.

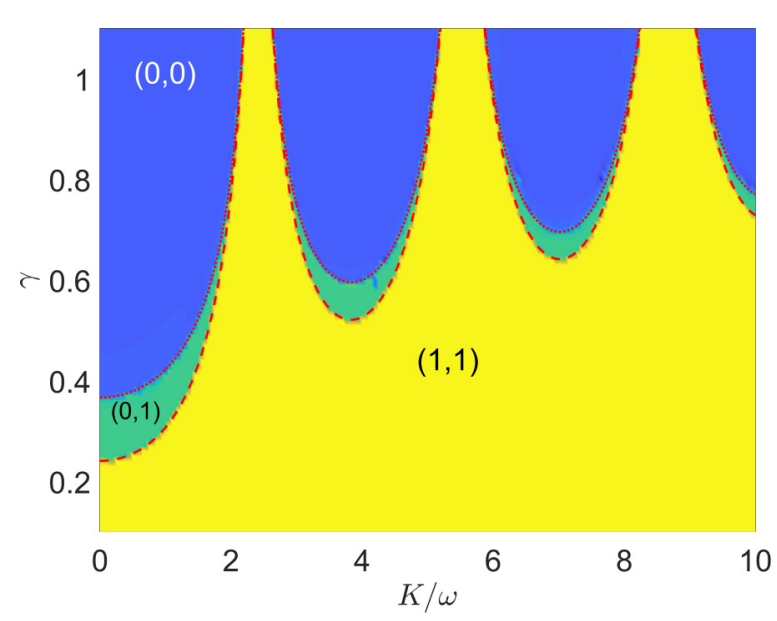

FIG. 6. The topological phase diagram of the non-Hermitian Floquet quasicrystal M4. System parameters are chosen as $\alpha=\frac{\sqrt{5}-1}{2}$, $J=2, V=1$, and the length of lattice is taken as $L=377$. Each region with a uniform color corresponds to a phase, whose winding numbers $\left(w_{1}, w_{2}\right)$ are denoted explicitly therein. The red dashed and dotted lines are phase boundaries satisfying Eqs. (39) and (40), respectively. 
In Fig. 6, we compute the winding numbers $\left(w_{1}, w_{2}\right)$ of M4 following their definitions in Table V. We observe that $\left(w_{1}, w_{2}\right)=(1,1)$ for the localized phase (below the red dashed line $),\left(w_{1}, w_{2}\right)=(0,1)$ for the mobility edge phase (between the red dashed and dotted lines), and $\left(w_{1}, w_{2}\right)=$ $(0,0)$ for the extended phase (above the red dotted line). These results confirm that the winding numbers $\left(w_{1}, w_{2}\right)$ could be utilized to distinguish quasicrystal phases of the nonHermitian Floquet Maryland model with different transport nature, and characterize the transitions between them. Notably, the winding numbers $\left(w_{1}, w_{2}\right)$ could also change with the increase of the ratio between the amplitude and frequency of the forcing $K / \omega$, which means that the topological properties of the non-Hermitian Maryland model can be engineered by the driving field.

\section{F. M5: Floquet spectrum, localization transition, mobility edge, and topological invariant}

To give another transparent view on how the mobility edge is modified by the driving field, we consider again a variant of the driven non-Hermitian AAH model, whose Hamiltonian in lattice representation takes the form

$$
\begin{aligned}
\hat{H}_{5}(t)= & \sum_{n} J\left(\hat{c}_{n}^{\dagger} \hat{c}_{n+1}+\text { H.c. }\right) \\
& +\sum_{n}\left[V /\left(1-\eta e^{i 2 \pi \alpha n}\right)-n K \cos (\omega t)\right] \hat{c}_{n}^{\dagger} \hat{c}_{n} .
\end{aligned}
$$

Here the parameter $\eta$ controls the amount of non-Hermiticity, and the system is quasiperiodic when $\alpha$ is irrational. In the high-frequency limit, we obtain the Floquet effective Hamiltonian of this M5 as

$$
\begin{aligned}
\hat{H}_{5 \mathrm{~F}}= & \sum_{n} J \mathcal{J}_{0}(K / \omega)\left(\hat{c}_{n}^{\dagger} \hat{c}_{n+1}+\text { H.c. }\right) \\
& +\sum_{n} V /\left(1-\eta e^{i 2 \pi \alpha n}\right) \hat{c}_{n}^{\dagger} \hat{c}_{n},
\end{aligned}
$$

where the hopping amplitude is controlled by the ratio between the amplitude and frequency of the driving force. Acting $\hat{H}_{5 \mathrm{~F}}$ on the state $|\psi\rangle=\sum_{n} \psi_{n} \hat{c}_{n}^{\dagger}|0\rangle$, we find the eigenvalue equation

$$
J_{\mathcal{J}}\left(\frac{K}{\omega}\right)\left(\psi_{n+1}+\psi_{n-1}\right)+\frac{V}{1-\eta e^{i 2 \pi \alpha n}} \psi_{n}=E \psi_{n},
$$

whose solutions yield the quasienergy $E$ and Floquet eigenstates of the system. Note that since the on-site potential amplitude $V_{n}=V_{-n}^{*}$, the system Hamiltonian also possesses the $\mathcal{P} \mathcal{T}$ symmetry and its spectrum could be real in certain parameter regime.

Following the method outlined in Ref. [87], the nonHermitian Floquet quasicrystal M5 is found to have a quasienergy-dependent mobility edge at $E=E_{c}$, where

$$
E_{c}=\left|J \mathcal{J}_{0}(K / \omega)(\eta+1 / \eta)\right| .
$$

Let us denote $E_{r}^{\max }$ and $E_{r}^{\min }$ as the maximum and minimum of the real parts of quasienergies over all states at a fixed set of system parameters. Then if $E_{r}^{\max }<E_{c}$, all eigenstates of the system are delocalized and the system sits in an extended phase. When $E_{r}^{\min }<E_{c}<E_{r}^{\max }$, we find the states
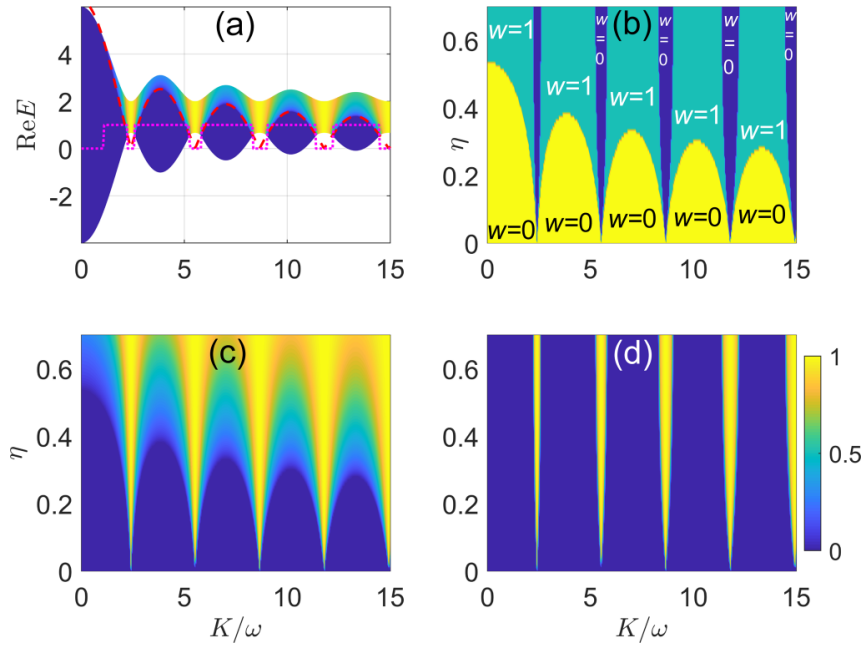

FIG. 7. The real part of quasienergies and IPRs of the nonHermitian Floquet quasicrystal M5. Other system parameters are set as $\alpha=\frac{\sqrt{5}-1}{2}, J=2.5, V=1$, and the length of lattice is taken as $L=610$. The color bars in (a), (c), and (d) are taken from zero to one. In (a), the real part of quasienergies $\operatorname{Re} E$ of all states are plotted with respect to $K / \omega$ at $\eta=0.5$, and the color of each state represents its IPR. The red dashed and magenta dotted lines show the mobility edge $E_{c}$ and winding number $w$ versus $K / \omega$. The phase diagram in (b) is obtained by evaluating $\operatorname{sign}\left(E_{c}-E_{r}^{\max }\right)+\operatorname{sign}\left(E_{c}-E_{r}^{\min }\right)$, which is equal to 2 (yellow regions), 0 (green regions), and -2 (blue regions) for the extended, mobility edge, and localized phases, respectively. The winding number is $w=1$ for the mobility edge phase and $w=0$ otherwise. The maximum and minimum of IPRs over all states are shown in (c) and (d), respectively. The mobility edge phase (localized phase) sets in when the maximum (minimum) of IPRs starts to deviate from zero.

with $\operatorname{Re} E<E_{c}\left(\operatorname{Re} E>E_{c}\right)$ to be extended (localized). A mobility edge thus emerges at $E=E_{c}$, and the system resides in the mobility edge phase under the condition $E_{r}^{\min }<E_{c}<$ $E_{r}^{\max }$. When $E_{c}<E_{r}^{\mathrm{min}}$, the system enters a phase in which all eigenstates are localized, and the mobility edge vanishes. By tuning the driving field parameters $K$ and $\omega$, the system could undergo alternate transitions among the extended, mobility edge and localized phases. The Floquet engineering scheme can again be utilized to dynamically control the mobility edges and transport nature of the non-Hermitian Floquet quasicrystal M5. We summarize our key results about this model in Table VI.

To demonstrate our theoretical findings, we compute the spectrum and IPRs of M5, as presented in Fig. 7. Note that in Fig. 7(b), we evaluated a winding number $w$ of the quasienergy with respect to the mobility edge $E_{c}$ (see Table VI for its definition), and find that $w=1$ for the mobility edge phase and $w=0$ otherwise. This winding number can thus be used as a topological order parameter to distinguish the mobility edge phase and other phases of the system, and to signify the transitions between them. The numerical results shown in Fig. 7 are all consistent with our theoretical predictions about the non-Hermitian Floquet quasicrystal M5. Combining these observations with the results obtained in Secs. III B-III E, we conclude that the Floquet engineering strategy could indeed be exploited as a useful means to control and modulate the 
TABLE VI. Summary of results for the non-Hermitian Floquet quasicrystal M5. $J$ is the nearest-neighbor hopping amplitude, $\eta$ controls the non-Hermiticity, $K$ is the driving amplitude, $\omega$ is the driving frequency, and $\mathcal{J}_{0}(K / \omega)$ denotes the Bessel function of first kind. $H_{5 \mathrm{~F}}(\theta)$ is obtained from $H_{5 \mathrm{~F}}$ after setting $V_{n}=V /\left[1-\eta e^{i(2 \pi \alpha n+\theta / L)}\right]$, with $V$ being the amplitude of on-site potential and $L$ the length of lattice. $E_{c}^{+}=E_{c}+i 0^{+}$is the base quasienergy with respect to which the winding number $w$ is defined.

\begin{tabular}{lccc}
\hline \hline Phase & Extended & Mobility Edge & Localized \\
\hline Condition & $E_{r}^{\max }<E_{c}$ & $E_{r}^{\min }<E_{c}<E_{r}^{\max }$ & $E_{c}<E_{r}^{\min }$ \\
IPR & $\simeq 0$ for all states & $>0 \& \simeq 0$ coexist & $>0$ for all states \\
Mobility edge & Real & $E_{c}=\left|J \mathcal{J}_{0}(K / \omega)(\eta+1 / \eta)\right|$ & Complex \\
Quasienergy & & $w=\int_{0}^{2 \pi} \frac{d \theta}{2 \pi i} \partial_{\theta} \ln \operatorname{det}\left[H_{5 F}(\theta)-E_{c}^{+}\right]=\left\{\begin{array}{l}1 \\
0\end{array}\right.$ & Mobility edge \\
Winding number & & & \\
\hline \hline
\end{tabular}

spectrum, topological, and transport properties in a broad class of non-Hermitian quasicrystals.

\section{SUMMARY AND DISCUSSION}

In this work, we apply Floquet driving fields to engineer $\mathcal{P} \mathcal{T}$ breaking, localization-to-delocalization, topological transitions, and mobility edges in non-Hermitian quasicrystals. Following the well-known scheme of dynamical localization, we utilize high-frequency shaking forces to control the hopping amplitudes of non-Hermitian quasiperiodic lattices, and observing alternate transitions between extended, localized, and mobility edge phases with the change of driving field parameters. We implement our scheme in five prototypical models of non-Hermitian quasicrystal, and obtain the conditions of their driving-assisted $\mathcal{P} \mathcal{T}$ transitions, localization-delocalization transitions, and mobility edges. We further introduced winding numbers of the quasienergy to distinguish non-Hermitian Floquet quasicrystals with different spectrum and localization features, and establishing the topological phase diagrams of these intriguing phases. Our results thus extend the study of non-Hermitian quasicrystals to periodically driven systems, and further uncover the usefulness of the Floquet engineering approach in the design and control of non-Hermitian quasicrystals with distinctive topological and transport properties.

In theory, the critical exponent of localization length (inverse Lyapunov exponent) could characterize the critical nature of delocalization-localization transitions [111]. For the original AAH model [see Eq. (15)] we have $\lambda=\ln (V / 2 J)$, and $\lambda^{-1} \propto\left|V-V_{c}\right|^{-1}$ around the critical point $V_{c}=2 \mathrm{~J}$ of the transition. The critical exponent is thus $v=1$. Referring to the Tables II-IV, we find that close to the critical points of models M1-M3, the localization length scales as $\lambda^{-1} \propto\left|V-V_{c}\right|^{-1}, \lambda^{-1} \propto\left|\gamma-\gamma_{c}\right|^{-1}$ and $\lambda^{-1} \propto \mid(K / \omega)-$ $\left.(K / \omega)_{c}\right|^{-1}$. Therefore, the critical exponent of these models is also $v=1$. In Ref. [111], it was found that for 1D systems with independent bond or site disorder, the critical exponent satisfies the Chayes' inequality $v \geqslant 2$. For $1 \mathrm{D}$ quasicrystals, since the disorder introduced by the quasiperiodic potential can be viewed as long-range correlated, the Chayes' inequality may not hold. Our results thus provide examples for the violation of the Chayes' inequality in 1D non-Hermitian Floquet quasicrystals.

Besides discrete lattices, localization transitions in quasicrystals have also been explored in continuum models
[112-114]. A generic observation for continuum models is that mobility edges will emerge beyond the tight-binding limit. Since we focused on the high-frequency region of the driving field in our study, the resulting systems are described by effective Floquet Hamiltonians with modified hopping amplitudes. Therefore, we expect that with long-range hoppings or in continuum versions of the models M1-M3, mobility edges may also appear, and the physical mechanism is similar to that discovered in Refs. [112-114]. In the meantime, the transition of the spectrum from real to complex in continuum versions of M1-M3 would be related to the transition from an extended or a localized phase to a mobility edge phase. Due to the possible presence of mobility edges, the energyindependent Lyapunov exponents shown in Tables II-IV may not be valid for continuum models, and should be reconsidered case by case.

In future work, it would be interesting to apply our scheme to the engineering of non-Hermitian quasicrystals with other types of mobility edges, long-range hoppings, many-body effects, and in higher spatial dimensions. In the present study, the experimentally established high-frequency approach [19] is applied to control the tunneling amplitudes, and we expect it to be valid under the condition listed below Eq. (9). Interestingly, our initial results (in preparation) suggest that driving fields could induce transitions among extended, localized, and mobility edge phases at generic frequencies. The results presented in Figs. 2-7 indicate that this general feature has already been captured qualitatively under the high-frequency approximation. Under moderate driving frequencies, quasicrystalline phases that are unique to nonHermitian Floquet systems may also emerge, which certainly deserve more thorough explorations.

\section{ACKNOWLEDGMENTS}

L.Z. is supported by the National Natural Science Foundation of China (Grant No. 11905211), the China Postdoctoral Science Foundation (Grant No. 2019M662444), the Fundamental Research Funds for the Central Universities (Grant No. 841912009), the Young Talents Project at Ocean University of China (Grant No. 861801013196), and the Applied Research Project of Postdoctoral Fellows in Qingdao (Grant No. 861905040009). 
[1] H. Lignier, C. Sias, D. Ciampini, Y. Singh, A. Zenesini, O. Morsch, and E. Arimondo, Dynamical Control of Matter-Wave Tunneling in Periodic Potentials, Phys. Rev. Lett. 99, 220403 (2007).

[2] F. L. Moore, J. C. Robinson, C. Bharucha, P. E. Williams, and M. G. Raizen, Observation of Dynamical Localization in Atomic Momentum Transfer: A New Testing Ground for Quantum Chaos, Phys. Rev. Lett. 73, 2974 (1994).

[3] M. Bitter and V. Milner, Experimental Observation of Dynamical Localization in Laser-Kicked Molecular Rotors, Phys. Rev. Lett. 117, 144104 (2016).

[4] M. C. Rechtsman, J. M. Zeuner, Y. Plotnik, Y. Lumer, D. Podolsky, F. Dreisow, S. Nolte, M. Segev, and A. Szameit, Photonic Floquet topological insulators, Nature (London) 496, 196 (2013).

[5] J. W. McIver, B. Schulte, F.-U. Stein, T. Matsuyama, G. Jotzu, G. Meier, and A. Cavalleri, Light-induced anomalous Hall effect in graphene, Nature Phys. 16, 38 (2020).

[6] S. Mukherjee and M. C. Rechtsman, Observation of Floquet solitons in a topological bandgap, Science 368, 856 (2020).

[7] A. Darabi, X. Ni, M. Leamy, and A. Alù, Reconfigurable Floquet elastodynamic topological insulator based on synthetic angular momentum bias, Sci. Adv. 6, eaba8656 (2020).

[8] W. Ma, L. Zhou, Q. Zhang, M. Li, C. Cheng, J. Geng, X. Rong, F. Shi, J. Gong, and J. Du, Experimental Observation of a Generalized Thouless Pump with a Single Spin, Phys. Rev. Lett. 120, 120501 (2018).

[9] G. Jotzu, M. Messer, R. Desbuquois, M. Lebrat, T. Uehlinger, D. Greif, and T. Esslinger, Experimental realization of the topological Haldane model with ultracold fermions, Nature (London) 515, 237 (2014).

[10] K. Wintersperger, C. Braun, F. N. Ünal, A. Eckardt, M. Di Liberto, N. Goldman, I. Bloch, and M. Aidelsburger, Realization of an anomalous Floquet topological system with ultracold atoms, Nature Phys. 16, 1058 (2020).

[11] A. Alberti, V. V. Ivanov, G. M. Tino, and G. Ferrari, Engineering the quantum transport of atomic wavefunctions over macroscopic distances, Nature Phys. 5, 547 (2009).

[12] C. Sias, H. Lignier, Y. P. Singh, A. Zenesini, D. Ciampini, O. Morsch, and E. Arimondo, Observation of Photon-Assisted Tunneling in Optical Lattices, Phys. Rev. Lett. 100, 040404 (2008).

[13] V. V. Ivanov, A. Alberti, M. Schioppo, G. Ferrari, M. Artoni, M. L. Chiofalo, and G. M. Tino, Coherent Delocalization of Atomic Wave Packets in Driven Lattice Potentials, Phys. Rev. Lett. 100, 043602 (2008).

[14] E. Haller, R. Hart, M. J. Mark, J. G. Danzl, L. Reichsöllner, and H.-C. Nägerl, Inducing Transport in a Dissipation-Free Lattice with Super Bloch Oscillations, Phys. Rev. Lett. 104, 200403 (2010).

[15] N. Poli, F.-Y. Wang, M. G. Tarallo, A. Alberti, M. Prevedelli, and G. M. Tino, Precision Measurement of Gravity with Cold Atoms in an Optical Lattice and Comparison with a Classical Gravimeter, Phys. Rev. Lett. 106, 038501 (2011).

[16] R. Ma, M. E. Tai, P. M. Preiss, W. S. Bakr, J. Simon, and M. Greiner, Photon-Assisted Tunneling in a Biased Strongly Correlated Bose Gas, Phys. Rev. Lett. 107, 095301 (2011).

[17] Y. Chen, S. Nascimbène, M. Aidelsburger, M. Atala, S. Trotzky, and I. Bloch, Controlling Correlated Tunneling and
Superexchange Interactions with ac-Driven Optical Lattices, Phys. Rev. Lett. 107, 210405 (2011).

[18] M. Grifoni and P. Hänggi, Driven quantum tunneling, Phys. Rep. 304, 229 (1998).

[19] A. Eckardt, Colloquium: Atomic quantum gases in periodically driven optical lattices, Rev. Mod. Phys. 89, 011004 (2017).

[20] M. Holthaus, Floquet engineering with quasienergy bands of periodically driven optical lattices, J. Phys. B: At. Mol. Opt. Phys. 49, 013001 (2016).

[21] G. Casati and L. Molinari, "Quantum chaos" with timeperiodic hamiltonians, Prog. Theor. Phys. Suppl. 98, 287 (1989).

[22] T. Oka and S. Kitamura, Floquet engineering of quantum materials, Annu. Rev. Condens. Matter Phys. 10, 387 (2019).

[23] J. Cayssol, B. Dóra, F. Simon, and R. Moessner, Floquet topological insulators, Phys. Status Solidi RRL 7, 101 (2013).

[24] F. Harper, R. Roy, M. S. Rudner, and S. L. Sondhi, Topology and broken symmetry in floquet systems, Annu. Rev. Condens. Matter Phys. 11, 345 (2020).

[25] M. S. Rudner and N. H. Lindner, Band structure engineering and non-equilibrium dynamics in Floquet topological insulators, Nature Rev. Phys. 2, 229 (2020).

[26] T. Kitagawa, Topological phenomena in quantum walks: elementary introduction to the physics of topological phases, Quant. Info. Proc. 11, 1107 (2012).

[27] K. Sacha and J. Zakrzewski, Time crystals: A review, Rep. Prog. Phys. 81, 016401 (2018).

[28] D. V. Else, C. Monroe, C. Nayak, and N. Y. Yao, Discrete time crystals, Ann. Rev. Condens. Matter Phys. 11, 467 (2020).

[29] L. Guo and P. Liang, Condensed matter physics in time crystals, New J. Phys. 22, 075003 (2020).

[30] V. Khemania, R. Moessner, and S. L. Sondhi, A brief history of time crystals, arXiv:1910.10745.

[31] S. Yao, Z. Yan, and Z. Wang, Topological invariants of Floquet systems: General formulation, special properties, and Floquet topological defects, Phys. Rev. B 96, 195303 (2017).

[32] R. Roy and F. Harper, Periodic table for Floquet topological insulators, Phys. Rev. B 96, 155118 (2017).

[33] F. Nathan and M. S. Rudner, Topological singularities and the general classification of Floquet-Bloch systems, New J. Phys. 17, 125014 (2015).

[34] R. W. Bomantara and J. Gong, Simulation of Non-Abelian Braiding in Majorana Time Crystals, Phys. Rev. Lett. 120, 230405 (2018).

[35] R. W. Bomantara and J. Gong, Quantum computation via Floquet topological edge modes, Phys. Rev. B 98, 165421 (2018).

[36] R. W. Bomantara and J. Gong, Measurement-only quantum computation with Floquet Majorana corner modes, Phys. Rev. B 101, 085401 (2020).

[37] E. J. Bergholtz, J. C. Budich, and F. K. Kunst, Exceptional topology of non-Hermitian systems, Rev. Mod. Phys. 93, 015005 (2021).

[38] Y. Ashida, Z. Gong, and M. Ueda, Non-Hermitian physics, Adv. Phys. 69, 249 (2020).

[39] V. M. Martinez Alvarez, J. E. Barrios Vargas, M. Berdakin, and L. E. F. Foa Torres, Topological states of non-Hermitian systems, Eur. Phys. J.: Spec. Top. 227, 1295 (2018).

[40] S. Yao and Z. Wang, Edge States and Topological Invariants of Non-Hermitian Systems, Phys. Rev. Lett. 121, 086803 (2018). 
[41] F. K. Kunst, E. Edvardsson, J. C. Budich, and E. J. Bergholtz, Biorthogonal Bulk-Boundary Correspondence in Non-Hermitian Systems, Phys. Rev. Lett. 121, 026808 (2018).

[42] L. Xiao, T. Deng, K. Wang, G. Zhu, Z. Wang, W. Yi, and P. Xue, Non-Hermitian bulk-boundary correspondence in quantum dynamics, Nature Phys. 16, 761 (2020).

[43] L. Zhou and J. Gong, Non-Hermitian Floquet topological phases with arbitrarily many real-quasienergy edge states, Phys. Rev. B 98, 205417 (2018).

[44] L. Zhou and J. Pan, Non-Hermitian Floquet topological phases in the double-kicked rotor, Phys. Rev. A 100, 053608 (2019).

[45] L. Zhou, Non-Hermitian Floquet topological superconductors with multiple Majorana edge modes, Phys. Rev. B 101, 014306 (2020).

[46] L. Zhou, Non-Hermitian Floquet phases with even-integer topological invariants in a periodically quenched two-leg ladder, Entropy 22, 746 (2020).

[47] J. Pan and L. Zhou, Non-Hermitian Floquet second order topological insulators in periodically quenched lattices, Phys. Rev. B 102, 094305 (2020).

[48] W. Zhao, L. Zhou, J. Liu, P. Tong, and K. Huang, Superexponential diffusion in nonlinear non-Hermitian systems, Phys. Rev. A 102, 062213 (2020).

[49] L. Zhou, Y. Gu, and J. Gong, Dual topological characterization of non-Hermitian Floquet phases, Phys. Rev. B 103, L041404 (2021).

[50] L. Zhou, Dynamical characterization of non-Hermitian Floquet topological phases in one dimension, Phys. Rev. B 100, 184314 (2019).

[51] M. S. Rudner and L. S. Levitov, Topological Transition in a Non-Hermitian Quantum Walk, Phys. Rev. Lett. 102, 065703 (2009).

[52] L. Xiao, X. Zhan, Z. Bian, K. Wang, X. Zhang, X. Wang, J. Li, K. Mochizuki, D. Kim, N. Kawakami, W. Yi, H. Obuse, B. C. Sanders and P. Xue, Observation of topological edge states in parity-time-symmetric quantum walks, Nature Phys. 13, 1117 (2017).

[53] X. Zhan, L. Xiao, Z. Bian, K. Wang, X. Qiu, B. C. Sanders, W. $\mathrm{Yi}$, and P. Xue, Detecting Topological Invariants in Nonunitary Discrete-Time Quantum Walks, Phys. Rev. Lett. 119, 130501 (2017).

[54] M. Li, X. Ni, M. Weiner, A. Alù, and A. B. Khanikaev, Topological phases and nonreciprocal edge states in non-Hermitian Floquet insulators, Phys. Rev. B 100, 045423 (2019).

[55] V. M. Vyas and D. Roy, Topological aspects of periodically driven non-hermitian su-schrieffer-heeger model, Phys. Rev. B 103, 075441 (2021).

[56] G. D. Valle and S. Longhi, Spectral and transport properties of time-periodic $\mathcal{P} \mathcal{T}$-symmetric tight-binding lattices, Phys. Rev. A 87, 022119 (2013).

[57] C. Yuce, PT symmetric Floquet topological phase, Eur. Phys. J. D 69, 184 (2015).

[58] R. de J. León-Montiel, M. A. Quiroz-Juárez, J. L. DomínguezJuárez, R. Quintero-Torres, J. L. Aragón, A. K. Harter, and Y. N. Joglekar, Observation of slowly decaying eigenmodes without exceptional points in Floquet dissipative synthetic circuits, Commun. Phys. 1, 88 (2018).

[59] J. Li, A. K. Harter, J. Liu, L. de Melo, Y. N. Joglekar, and L. Luo, Observation of parity-time symmetry breaking transi- tions in a dissipative Floquet system of ultracold atoms, Nature Commun. 10, 855 (2019).

[60] B. Höckendorf, A. Alvermann, and H. Fehske, Non-Hermitian Boundary State Engineering in Anomalous Floquet Topological Insulators, Phys. Rev. Lett. 123, 190403 (2019).

[61] D. Chowdhury, A. Banerjee, and A. Narayan, Light-driven Lifshitz transitions in non-Hermitian multi-Weyl semimetals, Phys. Rev. A 103, L051101 (2021).

[62] P. He and Z. Huang, Floquet engineering and simulating exceptional rings with a quantum spin system, Phys. Rev. A 102, 062201 (2020).

[63] E. N. Blose, Floquet topological phase in a generalized $\mathcal{P} \mathcal{T}$ symmetric lattice, Phys. Rev. B 102, 104303 (2020).

[64] S. Longhi, Floquet exceptional points and chirality in nonHermitian Hamiltonians, J. Phys. A: Math. Theor. 50, 505201 (2017).

[65] M. Chitsazi, H. Li, F. M. Ellis, and T. Kottos, Experimental Realization of Floquet $\mathcal{P} \mathcal{T}$-Symmetric Systems, Phys. Rev. Lett. 119, 093901 (2017).

[66] J. Gong and Q. Wang, Stabilizing non-Hermitian systems by periodic driving, Phys. Rev. A 91, 042135 (2015).

[67] Y. Cao, Y. Li, and X. Yang, Non-Hermitian bulk-boundary correspondence in a periodically driven system, Phys. Rev. B 103, 075126 (2021).

[68] H. Wu and J. An, Floquet topological phases of non-Hermitian systems, Phys. Rev. B 102, 041119(R) (2020).

[69] L. Ding, K. Shi, Q. Zhang, D. Shen, X. Zhang, and W. Zhang, Experimental Determination of $\mathcal{P} \mathcal{T}$-Symmetric Exceptional Points in a Single Trapped Ion, Phys. Rev. Lett. 126, 083604 (2021).

[70] X. Zhang and J. Gong, Non-Hermitian Floquet topological phases: Exceptional points, coalescent edge modes, and the skin effect, Phys. Rev. B 101, 045415 (2020).

[71] H. Wu, B. Wang, and J. An, Floquet second-order topological insulators in non-Hermitian systems, Phys. Rev. B 103, L041115 (2021).

[72] D. S. Ageev, A. A. Bagrov, and A. A. Iliasov, Deterministic chaos and fractal entropy scaling in Floquet conformal field theories, Phys. Rev. B 103, L100302 (2021).

[73] M. Bukov, L. D’Alessio, and A. Polkovnikov, Universal high-frequency behavior of periodically driven systems: From dynamical stabilization to Floquet engineering, Adv. Phys. 64, 139 (2015).

[74] N. Goldman and J. Dalibard, Periodically Driven Quantum Systems: Effective Hamiltonians and Engineered Gauge Fields, Phys. Rev. X 4, 031027 (2014).

[75] A. Eckardt and E. Anisimovas, High-frequency approximation for periodically driven quantum systems from a Floquet-space perspective, New J. Phys. 17, 093039 (2015).

[76] D. H. Dunlap and V. M. Kenkre, Dynamic localization of a charged particle moving under the influence of an electric field, Phys. Rev. B 34, 3625 (1986).

[77] K. Drese and M. Holthaus, Ultracold atoms in modulated standing light waves, Chem. Phys. 217, 201 (1997).

[78] K. W. Madison, M. C. Fischer, R. B. Diener, Qian Niu, and M. G. Raizen, Dynamical Bloch Band Suppression in an Optical Lattice, Phys. Rev. Lett. 81, 5093 (1998).

[79] S. Aubry and G. André, Analyticity breaking and Anderson localization in incommensurate lattices, Ann. Israel Phys. Soc. 3, 133 (1980). 
[80] P. G. Harper, Single band motion of conduction electrons in a uniform magnetic field, Proc. Phys. Soc. London A 68, 874 (1955).

[81] J. B. Sokoloff, Unusual band structure, wave function and electrical conductance in crystals with incommensurate periodic potentials, Phys. Rep. 126, 189 (1985).

[82] D. B. Grempel, S. Fishman, and B. E. Prange, Localization in an Incommensurate Potential: An Exactly Solvable Model, Phys. Rev. Lett. 49, 833 (1982).

[83] B. Simon, Almost periodic Schrödinger operators I V. The Maryland model, Ann. Phys. (NY) 159, 157 (1985).

[84] S. Longhi, Non-Hermitian Maryland model, Phys. Rev. B 103, 224206 (2021).

[85] S. Fishman, D. B. Grempel, and B. E. Prange, Chaos, Quantum Recurrences, and Anderson Localization, Phys. Rev. Lett. 49, 509 (1982).

[86] S. Ganeshan, K. Kechedzhi, and S. Das Sarma, Critical integer quantum Hall topology and the integrable Maryland model as a topological quantum critical point, Phys. Rev. B 90, 041405(R) (2014).

[87] T. Liu, H. Guo, Y. Pu, and S. Longhi, Generalized AubryAndré self-duality and mobility edges in non-Hermitian quasiperiodic lattices, Phys. Rev. B 102, 024205 (2020).

[88] S. Longhi, Topological Phase Transition in non-Hermitian Quasicrystals, Phys. Rev. Lett. 122, 237601 (2019).

[89] S. Longhi, Metal-insulator phase transition in a non-Hermitian Aubry-André-Harper model, Phys. Rev. B 100, 125157 (2019).

[90] S. Longhi, Phase transitions in a non-Hermitian Aubry-AndréHarper model, Phys. Rev. B 103, 054203 (2021).

[91] H. Jiang, L. Lang, C. Yang, S. Zhu, and S. Chen, Interplay of non-Hermitian skin effects and Anderson localization in nonreciprocal quasiperiodic lattices, Phys. Rev. B 100, 054301 (2019).

[92] Y. Liu, X.-P. Jiang, J. Cao, and S. Chen, Non-Hermitian mobility edges in one-dimensional quasicrystals with parity-time symmetry, Phys. Rev. B 101, 174205 (2020).

[93] Y. Liu, Y. Wang, Z. Zheng, and S. Chen, Exact non-Hermitian mobility edges in one-dimensional quasicrystal lattice with exponentially decaying hopping and its dual lattice, Phys. Rev. B 103, 134208 (2021).

[94] Y. Liu, Y. Wang, X. Liu, Q. Zhou, and S. Chen, Exact mobility edges, PT-symmetry breaking, and skin effect in onedimensional non-Hermitian quasicrystals, Phys. Rev. B 103, 014203 (2021)

[95] Z. Xu and S. Chen, Dynamical evolution in a one-dimensional incommensurate lattice with PT symmetry, Phys. Rev. A 103, 043325 (2021).

[96] Q. Zeng, S. Chen, and R. Lü, Anderson localization in the nonHermitian Aubry-André-Harper model with physical gain and loss, Phys. Rev. A 95, 062118 (2017).

[97] X. Cai, Boundary-dependent self-dualities, winding numbers, and asymmetrical localization in non-Hermitian ape- riodic one-dimensional models, Phys. Rev. B 103, 014201 (2021).

[98] A. Jazaeri and I. I. Satija, Localization transition in incommensurate non-Hermitian systems, Phys. Rev. E 63, 036222 (2001).

[99] Q. Zeng, Y. Yang, and R. Lü, Topological phases in onedimensional nonreciprocal superlattices, Phys. Rev. B 101, 125418 (2020).

[100] Q. Zeng, Y. Yang, and Y. Xu, Topological phases in nonHermitian Aubry-André-Harper models, Phys. Rev. B 101, 020201(R) (2020).

[101] L. Tang, G. Zhang, L. Zhang, and D. Zhang, Localization and topological transitions in non-Hermitian quasiperiodic lattices, Phys. Rev. A 103, 033325 (2021)

[102] T. Liu, S. Cheng, H. Guo, and X. Gao, Fate of Majorana zero modes, exact location of critical states, and unconventional real-complex transition in non-Hermitian quasiperiodic lattices, Phys. Rev. B 103, 104203 (2021).

[103] L. Zhai, G. Huang, and S. Yin, Cascade of the delocalization transition in a non-Hermitian interpolating Aubry-AndréFibonacci chain, Phys. Rev. B 104, 014202 (2021).

[104] Q. Zeng and Y. Xu, Winding numbers and generalized mobility edges in non-Hermitian systems, Phys. Rev. Res. 2, 033052 (2020).

[105] X. Xia, K. Huang, S. Wang, and X. Li, A new class of exact mobility edges in non-Hermitian quasiperiodic models, arXiv:2105.12640.

[106] L. Zhou and W. Han, Dimerized non-Hermitian quasicrystal, arXiv:2105.03302.

[107] Z. Xu, X. Xia, and S. Chen, Exact mobility edges and topological phase transition in two-dimensional non-Hermitian quasicrystal, arXiv:2106.11837.

[108] $\lambda<0$ here means that physically the Lyapunov exponents are not well-defined for extended states (with diverging localization lengths).

[109] A. Avila, Global theory of one-frequency Schrödinger operators, Acta. Math. 215, 1 (2015).

[110] S. Jitomirskaya and W. Liu, Arithmetic spectral transitions for the Maryland model, Commun. Pure Appl. Math. 70, 1025 (2017).

[111] J. T. Chayes, L. Chayes, Daniel S. Fisher, and T. Spencer, Finite-Size Scaling and Correlation Lengths for Disordered Systems, Phys. Rev. Lett. 57, 2999 (1986).

[112] R. B. Diener, G. A. Georgakis, J. Zhong, M. Raizen, and Q. Niu, Transition between extended and localized states in a onedimensional incommensurate optical lattice, Phys. Rev. A 64 033416 (2001).

[113] D. J. Boers, B. Goedeke, D. Hinrichs, and M. Holthaus, Mobility edges in bichromatic optical lattices, Phys. Rev. A 75, 063404 (2007).

[114] X. Li, X. Li, and S. Das Sarma, Mobility edges in onedimensional bichromatic incommensurate potentials, Phys. Rev. B 96, 085119 (2017). 